\title{
Computational analysis of functional SNPs in Alzheimer's disease-associated endocytosis genes
}

\author{
Han Jieh Tey ${ }^{1}$, Chong Han $\mathrm{Ng}^{\text {Corresp. } 1}$ \\ ${ }^{1}$ Faculty of Information Science and Technology, Multimedia University, Ayer Keroh, Melaka, Malaysia \\ Corresponding Author: Chong Han $\mathrm{Ng}$ \\ Email address: chng@mmu.edu.my
}

Background. From genome wide association studies on Alzheimer's disease (AD), it has been shown that many single nucleotide polymorphisms (SNPs) of genes of different pathways affect the disease risk. One of the pathways is endocytosis, and variants in these genes may affect their functions in amyloid precursor protein (APP) trafficking, amyloidbeta $(A \beta)$ production as well as its clearance in the brain. This study uses computational methods to predict the effect of novel SNPs, including untranslated region (UTR) variants, splice site variants, synonymous SNPs (sSNPs) and non-synonymous SNPs (nsSNPs) in three endocytosis genes associated with AD, namely PICALM, SYNJ1 and SH3KBP1. Materials and Methods. All the variants' information was retrieved from the Ensembl genome database, and then different variation prediction analyses were performed. UTRScan was used to predict UTR variants while MaxEntScan was used to predict splice site variants. Meta-analysis by PredictSNP2 was used to predict sSNPs. Parallel prediction analyses by five different software packages including SIFT, PolyPhen-2, Mutation Assessor, I-Mutant2.0 and SNPs\&GO were used to predict the effects of nsSNPs. The level of evolutionary conservation of deleterious nsSNPs was further analyzed using ConSurf server. Mutant protein structures of deleterious nsSNPs were modelled and refined using SPARKS-X and ModRefiner for structural comparison. Results. A total of 56 deleterious variants were identified in this study, including 12 UTR variants, 18 splice site variants, 8 sSNPs and 18 nsSNPs. Among these 56 deleterious variants, 7 variants were also identified in the Alzheimer's Disease Sequencing Project (ADSP), Alzheimer's Disease Neuroimaging Initiative (ADNI) and Mount Sinai Brain Bank (MSBB) studies. Discussion. The 56 deleterious variants were predicted to affect the regulation of gene expression, or have functional impacts on these three endocytosis genes and their gene products. The deleterious variants in these genes are expected to affect their cellular function in endocytosis and may be implicated in the pathogenesis of $A D$ as well. The biological consequences of these deleterious variants and their potential impacts on the disease risks could be further validated experimentally and may be useful for gene-disease 
association study. 
1 Computational analysis of functional SNPs in Alzheimer's disease-

2 associated endocytosis genes

3

4

5 Han Jieh Tey ${ }^{1}$, Chong Han $\mathrm{Ng}^{1}$

6

$7 \quad{ }^{1}$ Faculty of Information Science \& Technology, Multimedia University, Melaka, Malaysia.

8

9

10

11 Corresponding author:

12 Chong Han Ng

13 Multimedia University, Jalan Ayer Keroh Lama, 75450 Bukit Beruang, Melaka, Malaysia.

14 Email address: chng@mmu.edu.my 
15

\section{ABSTRACT}

Background. From genome wide association studies on Alzheimer's disease (AD), it has been shown that many single nucleotide polymorphisms (SNPs) of genes of different pathways affect the disease risk. One of the pathways is endocytosis, and variants in these genes may affect their functions in amyloid precursor protein (APP) trafficking, amyloid-beta (A $\beta$ ) production as well as its clearance in the brain. This study uses computational methods to predict the effect of novel SNPs, including untranslated region (UTR) variants, splice site variants, synonymous SNPs (sSNPs) and non-synonymous SNPs (nsSNPs) in three endocytosis genes associated with AD, namely PICALM, SYNJ1 and SH3KBP1.

Materials and Methods. All the variants' information was retrieved from the Ensembl genome database, and then different variation prediction analyses were performed. UTRScan was used to predict UTR variants while MaxEntScan was used to predict splice site variants. Meta-analysis by PredictSNP2 was used to predict sSNPs. Parallel prediction analyses by five different software packages including SIFT, PolyPhen-2, Mutation Assessor, I-Mutant2.0 and SNPs\&GO were used to predict the effects of nsSNPs. The level of evolutionary conservation of deleterious nsSNPs was further analyzed using ConSurf server. Mutant protein structures of deleterious nsSNPs were modelled and refined using SPARKS-X and ModRefiner for structural comparison.

Results. A total of 56 deleterious variants were identified in this study, including 12 UTR variants, 18 splice site variants, 8 sSNPs and 18 nsSNPs. Among these 56 deleterious variants, 7 variants were also identified in the Alzheimer's Disease Sequencing Project (ADSP), Alzheimer's Disease Neuroimaging Initiative (ADNI) and Mount Sinai Brain Bank (MSBB) studies.

Discussion. The 56 deleterious variants were predicted to affect the regulation of gene expression, or have functional impacts on these three endocytosis genes and their gene products. The deleterious variants in these genes are expected to affect their cellular function in endocytosis and may be implicated in the pathogenesis of AD as well. The biological consequences of these deleterious variants and their potential impacts on the disease risks could be further validated experimentally and may be useful for gene-disease association study. 


\section{Keywords}

44 SNP prediction; Alzheimer's disease; computational analysis; endocytosis genes; PICALM; 45 SYNJ1; SH3KBP1 
46

47

48

49

50

51

52

53

54

55

56

57

\section{INTRODUCTION}

Alzheimer's disease (AD) is the most common type of dementia. According to the amyloid hypothesis, amyloid-beta $(A \beta)$ is the primary factor to initiate a pathogenic cascade of $\mathrm{AD}$ in the brain (see review in $\mathrm{Du}$, Wang, \& Geng, (2018)). A $\beta$ is generated from proteolysis of its precursor, amyloid precursor protein (APP). The oligomeric assemblies of A $\beta$ that form amyloid plaques in extracellular space of neuron cell cause synaptic loss between neurons. Endocytosis is one of the biological processes affecting APP trafficking and many endocytosis genes, including BIN1, CD2AP, PICALM, EPHA1 and SORL1 were identified as AD-associated in GWAS and other genetic studies (see review in Giri, Zhang, \& Lü, (2016)).

From a study by Treusch et al. (2011), 40 types of $A \beta$ modifiers were identified through a yeast genome-wide overexpression study, which demonstrated a genetic link between $A \beta$ and endocytosis pathway in the pathogenesis of $\mathrm{AD}$. There are two types of $\mathrm{A} \beta$ modifiers, namely $A \beta$ suppressors, which decrease the toxicity of soluble $A \beta$ oligomers and $A \beta$ enhancers, which increase the toxicity. Among the identified $A \beta$ modifiers, three of the $A \beta$ suppressors are involved in endocytosis, namely YAP1802, INP52 and SLA1, and they have human orthologues, PICALM, SYNJ1 and SH3KBP1, respectively. Orthologous genes typically have similar biological function. Therefore, these three human orthologue genes may also play a role in modifying the toxicity of $\mathrm{A} \beta$ oligomers in human. Besides that, these human orthologues also play a role in clathrin-mediated endocytosis (CME), which is the major route for synaptic vesicle recycling (see review in Milosevic, (2018)). In addition, CME and its regulators have also been linked to the trafficking of $A \beta$ (Domínguez-Prieto et al., 2018). Therefore, it is of great interest to study the potential biological roles of PICALM, SYNJI and SH3KBPI genes in the pathogenesis of $\mathrm{AD}$.

PICALM (Phosphatidylinositol binding clathrin assembly protein) is mostly expressed in the brain capillary endothelium and neuron cells, and it is involved in cellular trafficking and the formation of clathrin-coated pit during CME (Tebar, Bohlander, \& Sorkin, 1999). PICALM gene is one of the validated AD risk genes first identified in a genome wide association study (GWAS) (Harold et al., 2009). The increased level of PICALM protein promotes the internalization of APP while its reduction causes the translocation of APP on the cell surface 
(Xiao et al., 2012). Besides that, PICALM helps in $A \beta$ clearance by increasing $A \beta$ translocation across the blood-brain barrier (Zhao et al., 2016). Several PICALM variants, including rs3851179 and rs592297, were identified to be associated with AD (Harold et al., 2009).

SYNJ1 (Synaptojanin 1) is the major synaptic polyphosphoinositide phosphatase, which regulates the uncoating and remobilization of synaptic vesicles during $\mathrm{CME}$ at the axon terminus (Cremona et al., 1999). SYNJ1 interacts with one of the AD risk factors, BIN1 (Bridging integrator 1) (Ramjaun et al., 1997). ApoE4-induced upregulation of SYNJ1 leads to the phospholipid homeostatic dysregulation in the brain, which is associated with the cognitive deficits in AD (Zhu et al., 2015). Conversely, downregulation of SYNJ1 not only restores the phospholipid homeostasis, but also reduces the levels of phosphorylated tau protein in vitro (Cao et al., 2017). On the other hand, variant in SYNJ1 gene was found to be associated with the heritable form of Parkinson's disease (PD) (Krebs et al., 2013).

SH3KBP1 (SH3-Domain Kinase Binding Protein 1), also known as CIN85, is an adaptor protein with multiple cell functions through binding to different protein partners. $S H 3 K B P 1$ is a homologous gene to one of the validated AD risk factors, CD2AP (see review in Rosenthal \& Kamboh, (2014)). These two homologous genes were reported to have high sequence and structural similarities, and they were identified as interacting partners (Mutso et al., 2018). A transgenic Drosophila study also showed the reduction of cindr gene expression, a fly orthologue of $S H 3 K B P 1$, increased tau neurotoxicity (Shulman et al., 2014).

Single nucleotide polymorphisms (SNPs) play important roles in many complex human diseases, including auto-immune diseases, diabetes and schizophrenia (see review in Visscher et al., (2017)). SNPs that fall on either coding or non-coding regions of a genome can exert their biological effects and could cause diseases. There are two types of SNPs in the coding regions, namely synonymous SNPs (sSNPs) and non-synonymous SNPs (nsSNPs). For sSNPs, they do not change the amino acid sequence of a translated protein. However, they can exert their biological consequences through changes in alternative splicing, mRNA stability or translation rate (see review in Sauna \& Kimchi-Sarfaty, (2011)). While for nsSNPs, these variants cause amino acids substitution and may have direct structural and functional impacts to the proteins. 
103 Non-coding regions SNPs may affect transcription factor binding, gene splicing, RNA

104 degradation and other biological processes.

105 Previous studies have demonstrated the important roles of genetic variants in the 106 development of AD. However, there are limited studies about the functional variants in these 107 AD-associated endocytosis genes, especially SYNJ1 and SH3KBP1 genes. In this study, the 108 effect of UTR variants, splice site variants, sSNPs and nsSNPs in PICALM, SYNJ1 and 109 SH3KBPl genes, was predicted and analyzed using computational methods. The variants with 110 high probability of affecting the protein structure and function are identified in the present study. 
111 Materials and methods

\section{Dataset retrieval}

113

114

115

116

117

118

119

120

121

122

123

124

125

126

127

128

129

130

131

132

133

134

135

The information for the genes of interest was retrieved from the Ensembl genome database (release 87) (http://www.ensembl.org/index.html) in March 2017 for computational analysis. Ensembl comprises the variant data from multiple sources, such as dbSNP, COSMIC and DVGa. The positions of the variants were mapped on the human reference genome GRCh38, which is also equivalent to the UCSC (University of California, Santa Cruz) hg38.

\section{Analysis of UTRs variants}

Both 3' and 5' UTR sequences of the genes of interest were collected from the curated database, UTRdb (http://utrdb.ba.itb.cnr.it/). These sequences with different variants were submitted to the UTRScan, which is a pattern matcher to search for UTR motifs collected in UTRSite (Grillo et al., 2010). UTRSite is a collection of functional sequence patterns located in 3 ' and 5' UTR sequences, such as upstream open reading frame (uORF), internal ribosome entry site (IRES), and polyadenylation signal (PAS). If different functional patterns are found between wild type and mutant UTR sequences, the variant is predicted to be functionally significant.

\section{Analysis of splice site variants}

The impact of splice site variants was predicted by using MaxEntScan integrated in Ensembl Variant Effect Predictor (VEP) (http://www.ensembl.org/Tools/VEP), which implements the Maximum Entropy Distribution approach. The input for the VEP requires only variant identifier (variant ID). For each variant, a consensus score with value in the range of -20 to +20 is calculated by MaxEntScan. Then, the score difference (in percentage) between wild type and mutant is calculated manually. The consensus score threshold of 3 and score difference threshold of $30 \%$ are applied to predict the splice site variants from both positive and negative controls (Desmet et al., 2009). Table S1 shows the possible consequences of splice site variants predicted by MaxEntScan. 
136

137

138

139

140

141

142

143

144

145

146

147

148

149

150

151

152

153

154

155

156

157

158

159

160

161

162

163

\section{Analysis of sSNPs}

PredictSNP2 (http://loschmidt.chemi.muni.cz/predictsnp2/) is a web server that predicts the effect of nucleotide substitution in any region of the genome. The server integrates five prediction tools - CADD, DANN, FATHMM, FunSeq2 and GWAVA (Bendl et al., 2016). The consensus score generated by PredictSNP2 ranges from -1 to +1 , in which the variant is considered neutral if the consensus score falls between -1 and 0 , and is deleterious if the consensus score falls between 0 and +1 .

\section{Analysis of nsSNPs}

Five prediction software packages that implement different prediction methodologies, namely SIFT (Ng \& Henikoff, 2001), PolyPhen-2 (Adzhubei, Jordan, \& Sunyaev, 2013), Mutation Assessor (Reva, Antipin, \& Sander, 2011), I-Mutant2.0 (Capriotti, Fariselli, \& Casadio, 2005) and SNPs\&GO (Capriotti et al., 2013), were used to predict the deleteriousness of all the nsSNPs. The nsSNPs that passed all the default cutoff points were considered to be deleterious nsSNPs. Then, the degree of evolutionary conservation of the deleterious nsSNPs was estimated using the ConSurf server (Ashkenazy et al., 2016), and the mutant protein structures were modelled and refined by SPARKS-X (Yang et al., 2011) and ModRefiner (Xu \& Zhang, 2011).

SIFT (Sorting Intolerant From Tolerant, version 5.2.2) from http://sift.jcvi.org/ is a sequence conservation-based package that predicts the functional impact of amino acid substitution based on sequence homology and physiochemical diversity (Ng \& Henikoff, 2001). The cutoff point was set at 0.05 , so variants with a SIFT score lower than 0.05 were considered deleterious.

PolyPhen-2 (Polymorphism phenotype 2, version 2.2.2) from

http://genetics.bwh.harvard.edu/pph2/, is a sequence- and structure-based prediction package. It predicts the impact of nsSNPs based on sequential and structural features collected from multiple databases and tools, such as UniProtKB, BLAST, Pfam and DSSP (Adzhubei et al., 2013). The cutoff point was set at 0.9, so variants with PolyPhen-2 score (known as PSIC score) higher than 0.9 were categorized as probably damaging. 

conservation-based prediction by assessing the entropy differences of an alignment position. It uses the sequence homology information of both protein families and subfamilies to assess the level of conservation of each residue (Reva et al., 2011). The functional impact of the variants was categorized into "neutral", "low", "medium" or "high" impact categories. In this study, the cutoff level for the deleterious variant was set at "medium" or "high" impact.

I-Mutant2.0 (http://folding.biofold.org/i-mutant/i-mutant2.0.html) is a structure analysisbased prediction software that analyzes the protein stability change for a single point variant (Capriotti et al., 2005). I-Mutant2.0 works by calculating the change in Gibbs free energy between the wild type and mutant protein structures. Variants with energy change value (DDG) less than -1 or greater than 1 indicate that these variants are destabilizing or stabilizing the protein, respectively. http://snps.biofold.org/snps-and-go/snps-and-go.html, combines both sequence and structure information to perform prediction. Information from Gene Ontology terms is also taken into consideration in predicting the functional impact of the variants (Capriotti et al., 2013). The cutoff point was set at 0.5 , so variants with probability greater than 0.5 were predicted as disease-related. analysis tools based on amino acid positions among the homologous protein sequences (Ashkenazy et al., 2016). In ConSurf, the conservation score on each protein residue indicates the level of conservation, with score " 1 " indicating the residue is highly variable and score "9" indicating the residue is highly conserved. Residues are also predicted to be buried (b), exposed (e), highly conserved and exposed (functional, f), or highly conserved and buried (structural, s).

SPARKS-X fold recognition server (http://sparks-lab.org/yueyang/server/SPARKS$\mathrm{X} /$ index.php) is a template-based protein structure modelling tool (Yang et al., 2011). The modelled protein structures were refined by using ModRefiner, which is a structure refinement server that improves the structure quality, in terms of side-chain positioning and backbone topology (Xu \& Zhang, 2011). The structural impacts of the nsSNPs can be predicted by 
193 studying the total free energy change between the wild type and mutant protein structures, as

194 well as the root mean square deviation (RMSD) of the mutant structures from the native protein 195 structure. 
196

197

198

199

200

201

202

203

204

205

206

207

208

209

210

211

212

213

214

215

216

217

218

219

220

221

\section{Results}

\section{Dataset retrieval from Ensembl}

The SNP datasets on PICALM, SYNJI and SH3KBP1 genes were retrieved from the Ensembl genome database. It contained a total of 1741 SNPs, including 395 UTRs (22\%) variants, 188 (11\%) splice site variants, 399 (23\%) sSNPs and 759 (43\%) nsSNP. Out of a total 759 nsSNPs for the genes of interest, 106 were classified as "deleterious" in SIFT and "probably damaging" in PolyPhen-2, according to the results in the Ensembl genome database. Figure S1 shows the total number of variants used in this study.

\section{Prediction analysis of UTRs variants}

The information of the retrieved UTRs sequence and the total number of variants on the corresponding region are shown in Table S2. There were no 5' UTR variants for SYNJ1 gene in the Ensembl genome database.

In the wild type UTR sequences of PICALM gene, a total of 11 signals were identified from the UTRScan. Out of a total of 35 UTR variants in PICALM gene, only one 3' UTR variant, rs796143140, caused an additional signal, namely K-box at position 212-219 of 3' UTR sequence. K-box is one of the 3' UTR motifs that mediates the negative post-transcriptional regulation of RNA (Ganguli et al., 2013). K-box motif was not present in the wild type UTR sequence of PICALM gene and the addition of the motif may affect transcript turnover and translational efficiency.

In the wild type 3' UTR sequence of SYNJ1 gene, a total of 20 signals were identified from UTRScan. Three 3' UTR variants, including rs777138831, rs147590143 and rs111516740, caused an addition of upstream open reading frame (uORF) while another four variants, including rs767649429, rs143739621, rs761852713 and rs79652470, resulted in a deletion of uORF in 3' UTR. Variant rs780084162 caused an additional signal named UNR-binding site (UNR-bs). UNR is one of the RNA-binding proteins that acts as a regulator in the initiation of internal ribosomal entry site (IRES)-mediated translation and cap-dependent translation (Saltel et 
222 al., 2017). Therefore, variant rs 780084162 may affect the expression of SYNJ1 gene and the

223 actual biological effect of the additional UNR-bs needs to be further experimentally validated.

224

225

226

227

228

229

230

231

232

233

234

235

236

237

238

239

240

241

242

243

244

245

246

247

248

249

For SH3KBP1 gene, there were a total of 21 signals identified in the wild type 3' and 5' UTR sequences. Variant rs192424738 caused an addition of uORF; variant rs755895016 caused a deletion of uORF in the 3' UTR sequence. Variant rs778448080 resulted in a deletion of GYbox at position 2064-2070 of the 3' UTR sequence. Like K-box and Brd-box, GY-box is one of the 3' UTR motifs that mediates the negative post-translational regulation of RNA (Lai, Tam, \& Rubin, 2005). Therefore, deletion of the GY-box caused by variant rs778448080 may affect the translation efficiency of $S H 3 K B P 1$ gene.

Table 1 lists the variants that change the number of motif matched in UTRSite, as compared with their wild type UTR sequences (see Table $S 3$ for complete prediction analysis results). Both 3' and 5' UTRs are enriched with cis-acting regulatory elements, and both UTRs are important in the regulation of protein expression. In this study, a total of 12 UTR variants were predicted to cause an addition or a deletion of regulatory elements in the UTR sequences. Further analysis on the impacts of these regulatory elements, including the effect of the miRNA binding to the UTR sequence is outside the scope of this study. However, it should be characterized in the future.

\section{Prediction analysis of splice site variants}

The prediction analysis of splice site variants was done using MaxEntScan, which is integrated in VEP. Submission to MaxEntScan requires only the SNP IDs. The consensus score and score difference between wild type and mutant sequences were obtained after the submission. The consensus score for each variant was calculated based on different proteincoding transcripts and the same variant may have different consensus scores on different transcripts. This allowed users to study the impact of the splicing variant on different transcripts. Table 2 shows the variants with score difference exceeding the defined threshold. Columns "MaxEntScan Ref" and "MaxEntScan Alt" indicate the wild type and mutant consensus scores, respectively. "Score difference" shows the differences between the wild type and mutant consensus scores (as percentages) to predict the variant effects in causing exon extension, intron 
250 retention or splice site creation. Table $S 4$ comprises the complete prediction analysis results for

251 all the splice site variants.

252 A total of 18 variants were predicted to either disrupt or create splice sites, including 3 ,

25313 and 2 variants from PICALM, SYNJ1 and SH3KBP1 genes, respectively. Among these

254 variants, 17 were predicted to disrupt the splice site, in which splicing was omitted or disrupted,

255 resulting in exon extension or intron retention. Variant rs533064963 in SYNJ1 gene was the only

256 variant predicted to create a new splice site in the intron.

257 The biological consequences of alternative splicing are frequently affected by genetic

258 variations. Alternative splicing also has been demonstrated as a key mechanism of the risk alleles

259 in genes including $C L U, P I C A L M$ and $P T K 2 B$ to exert their biological impacts in the

260 pathogenesis of $\mathrm{AD}$ (Raj et al., 2018). The 18 splice site variants that were predicted to cause

261 alternative splicing in this study should be further investigated for their structural and functional

262 impacts on the proteins.

263

264

265

266

267

268

269

270

271

272

273

274

275

276

\section{Prediction analysis of sSNPs}

Out of the 399 sSNPs we studied, eight sSNPs were predicted as deleterious sSNPs in PredictSNP2, including five from PICALM gene and three from SYNJ1 gene. Table 3 shows the eight deleterious sSNPs. Table S5-7 describe the complete prediction results of sSNPs in PICALM, SYNJ1 and SH3KBPI genes, respectively.

Among the 99 sSNPs in PICALM gene, five were predicted to have deleterious effects. Four out of those five, including rs779752380, rs777199260, rs775830584 and rs759002840, are located on the PICALM ANTH domain. Among the three deleterious SSNPs in SYNJ1 gene, only rs768565296 is located on the 5-phosphatase domain of the protein. For SH3KBPI gene, all the 112 sSNPs were predicted to have neutral effects on the gene.

sSNPs affect protein expression and conformation through the regulation of RNA splicing, mRNA stability, translation dynamics, translation rate and co-translational folding of the protein (Sauna \& Kimchi-Sarfaty, 2011). The actual biological impacts of the eight deleterious sSNPs predicted in this study should be validated in the future. 


\section{Prediction analysis of nsSNPs}

278

279

280

281

282

283

284

285

286

287

288

289

290

291

292

293

294

295

296

297

298

299

300

301

302

303

304

305

All 759 nsSNPs in the three genes were analyzed by five prediction packages. The Ensembl genome database contains prediction results from SIFT and PolyPhen-2, in which a total of 106 nsSNPs were predicted as "damaging" in SIFT and "probably damaging" in PolyPhen-2. Together with the prediction results from Mutation Assessor, I-Mutant2.0 and SNPs\&GO, a total of 18 nsSNPs exceeded the cutoff points of all five packages and were identified as deleterious nsSNPs. The prediction results of the deleterious nsSNPs are summarized in Table 4 while the complete prediction results of nsSNPs using five prediction packages are shown in Table S8. All the deleterious nsSNPs were predicted with high SIFT score and most of them have a PSIC score larger than 0.95 in PolyPhen-2. Prediction results from SIFT and PolyPhen-2 showed that all deleterious nsSNPs were highly conserved in the proteins (Table 4).

There were four deleterious nsSNPs in PICALM gene predicted in this study. All of them are located in amino acids 106 to 179 of the PICALM sequence. Positions 106 to 179 are located on the PICALM protein ANTH domain (23 to 283 aa) and ENTH domain (14 to 145 aa), and these two domains were reported to be functionally redundant (Maldonado-Baez et al., 2008; Manna et al., 2015). The ANTH domain of the protein is important in the binding to phosphatidylinositol 4,5-bisphosphate (PIP2). The binding of PICALM to PIP2 is important for the formation of clathrin-coated pit, which is one of the key functions of PICALM in CME (Ishikawa et al., 2015). These deleterious nsSNPs were predicted to cause conformation change and affect PICALM protein function. Figure 1 shows the sticks representation of the protein structural changes caused by the deleterious nsSNPs in PICALM gene. In Fig. 1, the variant residues are colored yellow while red dashed lines indicate the hydrogen bonds between the residues. Variants rs780443419 (F109S) and rs765338634 (L179P) resulted in an addition or a deletion of hydrogen bond formation between the mutant and neighboring amino acids. Therefore, the substitution of these protein residues could significantly affect the ANTH domain function as well as the overall PICALM protein structure.

For SYNJ1 gene, 13 nsSNPs were predicted as deleterious. Six of them including rs 781675993, rs398122403, rs762909719, rs771755243, rs768897710 and rs779479360 are 
306 located on the Sac domain while another four including rs756845805, rs751110096,

307 rs147929290 and rs745418083 are located on the 5-phosphatase domain. Both Sac domain and

308 5-phosphatase domain mediate the crucial SYNJ1 protein function of phosphate hydrolysis, and

309 they were reported to function in a concerted way (see review in Hsu \& Mao, (2013)). Besides

310 that, a previous study has shown that both Sac and 5-phosphatase domains are important in the

311 synaptic vesicle endocytosis and SYNJ1 D730A mutant completely eliminated the activity of 5-

312 phosphastase domain (Mani et al., 2007). Although the actual impact of the predicted deleterious

313 variants is yet to be determined, these variants may affect the domains function and influence the

314 role of SYNJ1 in endocytosis as well.

Variant rs770229859 is the only predicted deleterious nsSNP in SH3KBPl gene. This

variant changed residue arginine to glutamine at position 648, which is the C-terminal coiled-coil domain of the protein. The coiled-coil domain of SH3KBP1 was previously reported to be important in mediating the interaction between SH3KBP1 and phosphatidic acid (Zhang et al., 2009). Moreover, deletion of the coiled-coil domain directly affected the binding of SH3KBP1 to c-Cbl ubiquitin ligases, in which the interaction is essential for the initiation of CME regulated by SH3KBP1 (Zhang et al., 2009). Interestingly, another study has found that alanine mutation of four basic amino acids (K645, K646, R648 and R650) on the coiled-coil domain can completely disrupt the interaction of SH3KBP1 with phosphatidic acid and c-Cbl (Zheng, Zhang, \& Liao, 2014). Since the deleterious nsSNP rs770229859 resulted in amino acid substitution at position 648 of SH3KBP1, this variant is predicted to have a high likelihood of influencing the functions of the coiled-coil domain.

The structural and functional importance of the 18 deleterious nsSNPs were further analyzed using ConSurf analysis tools. Evolutionary conservation analysis determines the level of conservation of each protein residue and predicts the potential structural and functional importance of these deleterious variants to the protein. Figure 2 shows that 17 out of 18 (94\%) deleterious nsSNPs were analyzed to be "conserved", with 12 of them (70\%) "highly conserved" (score “9”) through homologous sequence alignment. Only one deleterious nsSNP, rs745418083 (L776S) in SYNJ1 gene, was estimated to be "intermediate" in terms of evolutionary conservation. Besides that, 11 of the $18(61 \%)$ deleterious variants were predicted as structural 
335 residues and the rest (39\%) were functional residues. Figure S2-4 shows the conservation scores

336 of full length proteins of PICALM, SYNJ1 and SH3KBP1, respectively.

337 Besides the prediction analysis of the functional and structural importance of the

338

339

340

341

342

343

344

345

346

347

348

349

350

351

352

353

354

355

356

357

358

359

360

361

362

363 deleterious nsSNPs and their level of conservation on the proteins, the changes of physical and chemical properties between wild type and mutant amino acids were studied. Table $S 9$ shows the hydropathy, polarity and charge differences between the wild type and mutant amino acids of the deleterious nsSNPs.

During protein synthesis, both covalent and non-covalent interactions are driven by the physical and chemical properties of the amino acids. The hydropathy, size and charge of the amino acids all play important roles in the protein synthesis (Biro, 2006). Table $S 9$ shows that the hydropathy in eight deleterious nsSNPs has changed from hydrophobic to hydrophilic, and the polarity of four nsSNPs has changed from non-polar to polar. The substitution of amino acids may affect both covalent and non-covalent interactions among amino acids, subsequently influencing the stability and conformation of protein structure.

To study the role of nsSNPs in affecting the total free energy and the stability of protein structures, full lengths of both wild type and mutant protein structures were modelled with the use of SPARKS-X server (Yang et al., 2011), and the structures were refined by using ModRefiner (Xu \& Zhang, 2011). Energy minimization of the protein structures was performed by GROMOS96 force field using Swiss-PdbViewer. Table 5 shows the energy deviation and RMSD between wild type protein structures of PICALM, SYNJ1 and SH3KBP1, and the 18 mutant protein structures of deleterious nsSNPs. RMSD values were calculated to provide an insight into the deviation between the wild type and mutant protein structures. The RMSD values are proportional to the deviation between the wild type and mutant structures. The total free energy of wild type PICALM structure was $-24238.746 \mathrm{~kJ} / \mathrm{mol}$, and the total free energy of the four PICALM mutant structures was marginally decreased. The RMSD values also showed a minor deviation ranging from 1.13 to $1.45 \AA$. For SYNJ1 protein, the total free energy of the wild type structure was $-19307.051 \mathrm{~kJ} / \mathrm{mol}$. The deviation of total free energy between SYNJ1 wild type and mutant protein structures is significant. Among the 13 SYNJ1 mutant structures, variant rs398122403 (R258Q) has the highest total free energy of $3057.503 \mathrm{~kJ} / \mathrm{mol}$, and variant 
$364 \mathrm{rs} 775515863(\mathrm{G} 487 \mathrm{R})$ has the lowest total free energy of $-28591.270 \mathrm{~kJ} / \mathrm{mol}$. Among all the 365 SYNJ1 variants, rs781675993 (N200K) has the highest RMSD value of $2.34 \AA$. Figure 3 shows 366 the structural comparison of the wild type SYNJ1 structure (green) and rs781675993 mutant

367 structure (blue). Superposition of the two structures shows the structural deviation caused by the 368 variant. The only SH3KBP1 mutant structure caused by variant rs770229859 (R648Q) showed a 369 decreased total free energy of $-16167.838 \mathrm{~kJ} / \mathrm{mol}$, with a RMSD value of $1.88 \AA$.

370

371

372

373

374

375

376

377

378

379

380

381

382

383

384

385

386

387

388

389

390

\section{Verification of nsSNP prediction principle}

To demonstrate the reliability of nsSNP prediction, we predicted the functional consequences of other nsSNPs that have been previously studied in other benchwork experiments. Ten PSEN1 pathogenic nsSNPs that have been validated experimentally to affect amyloid-beta (A $\beta$ ) level were retrieved from ALZFORUM (https://www.alzforum.org/). Besides that, another five PSEN1 nsSNPs, including three non-pathogenic variants and two variants that have never been reported to be deleterious or disease-associated, were selected as negative controls. The prediction results of the total 15 nsSNPs in PSEN1 gene are shown in Table S10. The prediction results show that nine out of these ten pathogenic nsSNPs were predicted deleterious by all five prediction packages used in this paper. The only pathogenic nsSNP that was not predicted as deleterious variant, which is rs63750231 (E280A), has I-Mutant DDG and SNPs\&GO score that is lower than the cutoff point. All these five negative controls of PSEN1 nsSNPs were predicted to be non-deleterious to the protein.

Computational prediction of the biological consequences of variants was previously reported to yield a high false positive (FP) and low false negative (FN) rate, suggesting that computational prediction tends to overestimate the deleterious effects of variants (Miosge et al., 2015). To reduce the FP rate, our study implemented meta-prediction method to perform functional prediction of nsSNPs. Despite one FN prediction result (rs63750231) was reported, the overall prediction results of the PSEN1 pathogenic and non-pathogenic nsSNPs indicate that the prediction approaches which were used, reliably predicted the functional consequences of nsSNPs. 
391

392

393

394

395

396

397

398

399

400

401

402

403

404

405

406

407

408

409

410

411

412

413

414

415

416

\section{NGS data of AD studies}

To check if any of the 56 deleterious variants were identified in the next generation sequencing (NGS) studies of AD, genotype calling data (variant calling format (.VCF) files) were retrieved from three studies including Alzheimer's Disease Sequencing Project (ADSP), Alzheimer's Disease Neuroimaging Initiative (ADNI) and Mount Sinai Brain Bank (MSBB) studies. ADSP aims to identify genetic variants that increase the risk for or protect against AD. The study consists of two parts, including a whole genome sequencing (WGS) of 584 samples family-based study and a whole exome sequencing (WES) of 10,061 samples case-control study (Beecham et al., 2017). Genotype calling data of both WGS and WES were retrieved from the study. For ADNI, the study is primarily designed to discover novel biomarkers from a total of 800 individuals for the early detection of AD. The 800 individuals consist of 200 elderly controls, 400 mild cognitive impairment (MCI) and 200 AD patients. WGS of the 800 individuals was performed in the following add-on study and the genotype calling data is publicly available (Weiner et al., 2015). For MSBB study, it aims to discover novel molecular mechanism of $\mathrm{AD}$ pathogenesis from genomic, transcriptomic and proteomic data consisting of 364 AD case-control human brains (Wang et al., 2018). The raw and processed data of MSBB study are available through the Synapse software platform (https://www.synapse.org/\#!Synapse:syn3159438) and the .VCF file of WES of the study was retrieved.

Among the 56 deleterious variants predicted in this study, seven variants were also identified in ADSP, ADNI or MSBB studies. Table S11 shows the deleterious variants that were identified in the NGS studies of AD. Identification of these deleterious variants in these NGS studies further suggests their potential association with AD. In these studies, the genotype data was mapped on the reference genome GRCh37 while the variant data retrieved from Ensembl genome database was mapped on GRCh38. Therefore, the variant positions in both GRCh37 and GRCh38 are provided in Table S11. 


\section{Discussion}

418

419

420

421

422

423

424

425

426

427

428

429

430

431

432

433

434

435

436

437

438

439

440

441

442

443

444

445

446

In this study, a total of 56 SNPs in PICALM, SYNJ1 and SH3KBP1 genes were predicted as deleterious variants for the genes and their gene products. These deleterious variants included 12 UTR variants, 18 splice site variants, 8 sSNPs and 18 nsSNPs. These 12 deleterious UTR variants were predicted to cause an addition or a deletion of cis-acting elements in UTR sequences, which may affect the post-transcriptional regulation of the genes. These 18 deleterious splice site variants were predicted to disrupt gene splicing, which can produce truncated or extended forms of the proteins. Meta-analysis by PredictSNP2 has predicted a total of 8 deleterious sSNPs in PICALM and SYNJ1 genes. These deleterious UTR variants, splice site variants and sSNPs were expected to affect the regulation of gene expression, as well as the stability of the mRNAs. For the 18 deleterious nsSNPs, most of them are located on the functional domains of the proteins. These domains mediate the main protein functions in the regulation of clathrin-mediated endocytosis (CME). ANTH domain on PICALM regulates the formation of clathrin-coated pit while coiled-coil domain on SH3KBP1 has an important role in the initiation of CME (Ishikawa et al., 2015; Zhang et al., 2009). Specifically, both of the domains are involved in the early stage of CME. For SYNJ1, the hydrolysis of PI(4,5)P2 by Sac domain and 5-phosphatase domain may be involved in the disassembly of clathrin-coated vesicles (Hsu \& Mao, 2013), which is in the late stage of CME. Therefore, identification of deleterious nsSNPs on these functional domains may not only help to elucidate the functional impacts of these variants to the proteins, but also may gain insight into the biological roles of these domains in CME. Moreover, three endocytosis genes in this study have been linked to the pathogenesis of $\mathrm{AD}$. Hence, the prediction analysis of the deleterious variants may be relevant for the gene-disease association study.

To date, GWAS and other genetic studies have successfully identified more than 20 risk genes associated with $\mathrm{AD}$, but the molecular mechanisms of these genes remain to be determined. To understand the molecular basis of these AD-associated genes in the disease, one of the approaches is to identify functional SNPs from a vast number of neutral SNPs in the gene of interest. However, it was estimated that SNP occurs in every 100 to 300 bases and there are up to ten million SNPs in the entire human genome (Ke, Taylor, \& Cardon, 2008). The massive number of SNPs in the entire human genome makes it impractical to perform laboratory 
447 experiments to determine the functional consequences of all the SNPs, even for a single gene.

448 For that reason, computational methods become an alternative and important way to prioritize

449 the SNPs that are possibly structurally or functionally significant for the genes of interest.

450 Computational methods such as prediction and modelling tools allow researchers to identify

451 functionally significant SNPs from neutral SNPs. The prediction accuracy is expected to be

452 improved when results from multiple algorithms are combined to perform meta-prediction.

453 Besides that, computational methods are able to provide high throughput prediction results at

454 lower cost and faster time, as compared with laboratory experiments. Although study by Miosge

455 et al. (2015) has pointed out the low specificity of computational prediction, the discrepancy

456 between the prediction and experimental results of the variants impacts can be further improved

457 by providing a better data quality and input alignment design during computational prediction

458 (Gallion et al., 2017). In conclusion, computational methods is an essential part to provide initial

459 assessment and prioritize the SNPs that are most likely to be functional, which can largely

460 improve research efficiency.

461

462

463

464

465

466

467

468

469

470

471

472

473

474

475

476

477

Although computational methods can significantly contribute to the task of identifying functional SNPs, a limited number of studies had performed function prediction analysis of the variants located in the disease-associated genes. Moreover, most of the function prediction studies of variants only focus on annotating the functional consequences and disease-relatedness of nsSNP since it was conventionally thought to have a higher impact on the phenotype rather than the other types of variations. However, nsSNPs, sSNPs and 5' UTR variants have been reported to share similar likelihoods of association in Mendelian diseases (Chen et al., 2010). Other than these three types of variants, more than 18,000 of all variants reported in the Human Gene Mutation Database (HGMD) are splicing variants, which is about $9 \%$ of the total number of variants in the database (Stenson et al., 2017). In the pathogenesis of complex diseases, the roles of genetic factors are much more complicated than those in Mendelian diseases. Genetic variants in non-coding regions may play a more prominent role than in coding regions in complex diseases since most of the disease-associated variants identified in GWAS are located in non-coding regions (Zhang \& Lupski, 2015). Identifying pathogenic variants in both coding and non-coding regions of genes associated with complex diseases can help to explain the genetics factors and networks of the diseases. Therefore, this study does not target nsSNPs exclusively because it may overlook other functionally significant variants that are associated with AD. 
To our knowledge, 55 out of the 56 deleterious variants predicted in this study were

479 reported for the first time to be functionally or structurally significance for PICALM, SYNJ1 and

480 SH3KBPl genes, and their gene products. Only one deleterious variant, which is rs 398122403

481 (R258Q) in SYNJ1 gene, was previously reported to be associated with Parkinson's disease (PD)

482 (Krebs et al., 2013). Variant rs398122403 was found to disrupt the phosphatase activity of Sac

483 domain on SYNJ1 protein, leading to an impaired synaptic function of SYNJ1 and causing an

484 accumulation of endocytic proteins in the brains of mutant mice, developing neurological

485 symptoms similar to PD patients (Cao et al., 2017). Although AD and PD have different clinical

486 and pathological symptoms, many studies suggested that they share some common molecular

487 mechanisms, such as oxidative stress and mitochondrial dysfunction (see review in Tan et al.,

488 (2019)). However, in the case of SYNJ1, several studies suggested that the downregulation of

489 SYNJ1 may alleviate the pathogenesis of AD (McIntire et al., 2012; Zhu et al., 2013).

490 Intriguingly, the biological effects of the SYNJ1 in AD don't seem to be in line with budding

491 yeast and C. elegans orthologues (Treusch et al., 2011). Since the validated PD-associated

492 variant rs398122403 was also predicted as deleterious variant in this study, and the mutant

493 protein structure of this variant has the highest total free energy among all the deleterious

494 variants, the prediction results of this study may serve as a good target for further investigation.

495 Nevertheless, it is not known if the variant rs398122403 is also associated with AD. SYNJ1 is

496 one of very few proteins associated with both AD and PD. Therefore, the SYNJ1 variants

497 predicted in this study may be useful to discover the biological roles of SYNJ1 on the molecular

498 mechanisms in AD and PD.

Previously, several AD-associated common variants have been identified from GWAS.

500 For example, variants rs3851179, rs541458 and rs561655 are associated with AD. These three

501 variants were not identified in this study since they are intergenic variants which are located

502 upstream of PICALM gene. On the other hand, the minor allele frequency (MAF) of these three

503 SNPs are over 0.3, which is much higher as compared to the deleterious variants predicted in this

504 study. Previous studies have reported that most of the AD-associated common variants have a

505 smaller effect size on the disease risk as compared to rare variants (see review in Nicolas,

506 Charbonnier, \& Campion, (2016)). Therefore, the current research direction is gearing more

507 towards to identifying rare variants with a larger effect size that are located in different genes

508 (Pierre \& Genin, 2014). The MAF of the 56 deleterious variants predicted in this study are 
509 provided in Tables 1, 2, 3 and 4. The MAF data was obtained from the Exome Aggregation

510 Consortium (ExAC) in aggregated populations. All the deleterious variants are very rare variants

511 (average MAF < 0.00002). According to the "common disease common variant" (CDCV)

512 hypothesis, the risk of getting a common disease would be relatively high along with the

513 appearance of disease-predisposing alleles. Assuming that the common alleles are the actual

514 cause of common diseases, many causative alleles would have been identified through GWAS.

515 However, the current data do not seem to support this hypothesis since only a very limited

516 number of variants have been successfully linked to the pathogenesis of certain diseases.

517 Moreover, common variants identified from GWAS are insufficient to explain the missing

518 heritability of complex phenotypes and diseases (see review in Blanco-Gómez et al., (2016)). On

519 the other hand, the rare variants absent in GWAS have been suggested to be an important factor

520 in common disease susceptibility in the common disease rare variant (CDRV) hypothesis (Auer

$521 \&$ Lettre, 2015). Despite different strategies used in the identification of disease-associated

522 variants in both hypotheses, they can work together to identify the common variants along with

523 the genomic region and locus through GWAS, followed by conducting the functional analysis of

524 the rare variants. Currently, no AD-associated rare variants in PICALM, SYNJ1 and SH3KBP1

525 genes has been reported by other studies so far. It is plausible that the sample size of GWAS

526 might not be sufficient to identify disease-associated rare variants. Hence, our prediction analysis

527 may be useful to identify functional variants which could have been absent in GWAS due to their

528 rare MAF. 


\section{CONCLUSION}

530 In our study, a total of 56 rare variants in PICALM, SYNJ1 and SH3KBPI genes were 531 predicted as deleterious variants. These deleterious variants were predicted to affect the

532 regulation of gene expression and protein functions. Three of these genes have cellular functions 533 involved in clathrin-mediated endocytosis (CME) and deleterious variants in these genes were 534 expected to affect the functions of these proteins in endocytosis. Moreover, these genes were 535 previously reported as AD-associated and they are implicated in the pathogenesis of AD. The 536 deleterious variants in these genes may affect AD pathogenesis through the disruption of the 537 gene expression and protein functions. In addition, seven of the deleterious variants were also 538 identified in three large NGS studies of AD, further suggesting their possible roles in AD 539 pathogenesis. The actual roles of these deleterious variants in affecting protein functions in $540 \mathrm{CME}$, as well as their implications in $\mathrm{AD}$, are recommended to be validated by future laboratory 541 experiments. 


\section{ACKNOWLEDGEMENT}

543 We would like to show our gratitude to Dr. Henry Lee Seldon and Dr. Vachiranee 544 Limviphuvadh for their valuable opinions to improve the manuscript. 
545

546

547

548

549

550

551

552

553

554

555

556

557

558

559

560

561

562

563

564

565

566

567

568

569

570

571

572

573

574

575

576

577

578

579

580

581

582

583

584

585

586

587

588

589

Adzhubei, I., Jordan, D. M., \& Sunyaev, S. R. (2013). Predicting Functional Effect of Human Missense Mutations Using PolyPhen-2. Current Protocols in Human Genetics, 76(1), 7.20.1-7.20.41. https://doi.org/10.1002/0471142905.hg0720s76

Ashkenazy, H., Abadi, S., Martz, E., Chay, O., Mayrose, I., Pupko, T., \& Ben-Tal, N. (2016). ConSurf 2016: an improved methodology to estimate and visualize evolutionary conservation in macromolecules. Nucleic Acids Research, 44(W1), W344-W350. https://doi.org/10.1093/nar/gkw408

Auer, P. L., \& Lettre, G. (2015). Rare variant association studies: Considerations, challenges and opportunities. Genome Medicine. https://doi.org/10.1186/s13073-015-0138-2

Beecham, G. W., Bis, J. C., Martin, E. R., Choi, S.-H., DeStefano, A. L., van Duijn, C. M., ... Schellenberg, G. (2017). The Alzheimer's Disease Sequencing Project: Study design and sample selection. Neurology. Genetics, 3(5), e194. https://doi.org/10.1212/NXG.0000000000000194

Bendl, J., Musil, M., Štourač, J., Zendulka, J., Damborský, J., \& Brezovský, J. (2016). PredictSNP2: A Unified Platform for Accurately Evaluating SNP Effects by Exploiting the Different Characteristics of Variants in Distinct Genomic Regions. PLOS Computational Biology, 12(5), e1004962. https://doi.org/10.1371/journal.pcbi.1004962

Biro, J. C. (2006). Amino acid size, charge, hydropathy indices and matrices for protein structure analysis. Theoretical Biology \& Medical Modelling, 3, 15. https://doi.org/10.1186/17424682-3-15

Blanco-Gómez, A., Castillo-Lluva, S., del Mar Sáez-Freire, M., Hontecillas-Prieto, L., Mao, J. H., Castellanos-Martín, A., \& Pérez-Losada, J. (2016). Missing heritability of complex diseases: Enlightenment by genetic variants from intermediate phenotypes. BioEssays, 38(7), 664-673. https://doi.org/10.1002/bies.201600084

Cao, M., Wu, Y., Ashrafi, G., McCartney, A. J., Wheeler, H., Bushong, E. A., ... De Camilli, P. (2017). Parkinson Sac Domain Mutation in Synaptojanin 1 Impairs Clathrin Uncoating at Synapses and Triggers Dystrophic Changes in Dopaminergic Axons. Neuron, 93(4), 882896.e5. https://doi.org/10.1016/j.neuron.2017.01.019

Capriotti, E., Calabrese, R., Fariselli, P., Martelli, P. L., Altman, R. B., \& Casadio, R. (2013). WS-SNPs\&GO: a web server for predicting the deleterious effect of human protein variants using functional annotation. BMC Genomics, 14 Suppl 3(Suppl 3), S6. https://doi.org/10.1186/1471-2164-14-S3-S6

Capriotti, E., Fariselli, P., \& Casadio, R. (2005). I-Mutant2.0: predicting stability changes upon mutation from the protein sequence or structure. Nucleic Acids Research, 33(Web Server), W306-W310. https://doi.org/10.1093/nar/gki375

Chen, R., Davydov, E. V., Sirsota, M., \& Butte, A. J. (2010). Non-synonymous and synonymous coding SNPS show similar likelihood and effect size of human disease association. PLoS ONE, 5(10), 1-6. https://doi.org/10.1371/journal.pone.0013574

Cremona, O., Di Paolo, G., Wenk, M. R., Luthi, A., Kim, W. T., Takei, K., ... De Camilli, P. (1999). Essential role of phosphoinositide metabolism in synaptic vesicle recycling. Cell, 99(2), 179-188. https://doi.org/S0092-8674(00)81649-9 [pii]

Desmet, F.-O., Hamroun, D., Lalande, M., Collod-Béroud, G., Claustres, M., \& Béroud, C. (2009). Human Splicing Finder: an online bioinformatics tool to predict splicing signals. Nucleic Acids Research, 37(9), e67-e67. https://doi.org/10.1093/nar/gkp215

Peer) reviewing PDF | (2019:03:35860:1:1:NEW 11 Jul 2019) 
590

591

592

593

594

595

596

597

598

599

600

601

602

603

604

605

606

607

608

609

610

611

612

613

614

615

616

617

618

619

620

621

622

623

624

625

626

627

628

629

630

631

632

633

634

635

Domínguez-Prieto, M., Velasco, A., Tabernero, A., \& Medina, J. M. (2018). Endocytosis and Transcytosis of Amyloid- $\beta$ Peptides by Astrocytes: A Possible Mechanism for Amyloid- $\beta$ Clearance in Alzheimer's Disease. Journal of Alzheimer's Disease, 65(4), 1109-1124. https://doi.org/10.3233/JAD-180332

Du, X., Wang, X., \& Geng, M. (2018). Alzheimer's disease hypothesis and related therapies. Translational Neurodegeneration, 7(1), 2. https://doi.org/10.1186/s40035-018-0107-y

Gallion, J., Koire, A., Katsonis, P., Schoenegge, A. M., Bouvier, M., \& Lichtarge, O. (2017). Predicting phenotype from genotype: Improving accuracy through more robust experimental and computational modeling. Human Mutation. https://doi.org/10.1002/humu.23193

Ganguli, S., Das, S. G., Chakraborty, H. J., Gupta, S., \& Datta, A. (2013). Identification of regulatory sequence signatures in microRNA precursors implicated in neurological disorders. Advances in Bioscience and Biotechnology, 04(05), 26-33. https://doi.org/10.4236/abb.2013.45A003

Giri, M., Zhang, M., \& Lü, Y. (2016). Genes associated with Alzheimer's disease: an overview and current status. Clinical Interventions in Aging, 11, 665-681. https://doi.org/10.2147/CIA.S105769

Grillo, G., Turi, A., Licciulli, F., Mignone, F., Liuni, S., Banfi, S., .. Pesole, G. (2010). UTRdb and UTRsite (RELEASE 2010): a collection of sequences and regulatory motifs of the untranslated regions of eukaryotic mRNAs. Nucleic Acids Research, 38(suppl_1), D75D80. https://doi.org/10.1093/nar/gkp902

Harold, D., Abraham, R., Hollingworth, P., Sims, R., Gerrish, A., Hamshere, M. L., ... Williams, J. (2009). Genome-wide association study identifies variants at CLU and PICALM associated with Alzheimer's disease. Nature Genetics, 41(10), 1088-1093. https://doi.org/10.1038/ng.440

Hsu, F. S., \& Mao, Y. (2013). The Sac domain-containing phosphoinositide phosphatases: Structure, function, and disease. Frontiers in Biology. https://doi.org/10.1007/s11515-0131258-y

Ishikawa, Y., Maeda, M., Pasham, M., Aguet, F., Tacheva-Grigorova, S. K., Masuda, T., ... Maeda, T. (2015). Role of the clathrin adaptor PICALM in normal hematopoiesis and polycythemia vera pathophysiology. Haematologica. https://doi.org/10.3324/haematol.2014.119537

Ke, X., Taylor, M. S., \& Cardon, L. R. (2008). Singleton SNPs in the human genome and implications for genome-wide association studies. European Journal of Human Genetics. https://doi.org/10.1038/sj.ejhg.5201987

Krebs, C. E., Karkheiran, S., Powell, J. C., Cao, M., Makarov, V., Darvish, H., ... Paisán-Ruiz, C. (2013). The Sac1 Domain of SYNJ1 Identified Mutated in a Family with Early-Onset Progressive Parkinsonism with Generalized Seizures. Human Mutation, 34(9), 1200-1207. https://doi.org/10.1002/humu.22372

Lai, E. C., Tam, B., \& Rubin, G. M. (2005). Pervasive regulation of Drosophila Notch target genes by GY-box-, Brd-box-, and K-box-class microRNAs. Genes \& Development, 19(9), 1067-1080. https://doi.org/10.1101/gad.1291905

Maldonado-Baez, L., Dores, M. R., Perkins, E. M., Drivas, T. G., Hicke, L., \& Wendland, B. (2008). Interaction between Epsin/Yap180 Adaptors and the Scaffolds Ede1/Pan1 Is Required for Endocytosis. Molecular Biology of the Cell. https://doi.org/10.1091/mbc.E0710-1019 
636

637

638

639

640

641

642

643

644

645

646

647

648

649

650

651

652

653

654

655

656

657

658

659

660

661

662

663

664

665

666

667

668

669

670

671

672

673

674

675

676

677

678

679

680

681

Manna, P. T., Gadelha, C., Puttick, A. E., \& Field, M. C. (2015). ENTH and ANTH domain proteins participate in AP2-independent clathrin-mediated endocytosis. Journal of Cell Science. https://doi.org/10.1242/jcs. 167726

McIntire, L. B. J., Berman, D. E., Myaeng, J., Staniszewski, A., Arancio, O., Di Paolo, G., \& Kim, T.-W. (2012). Reduction of synaptojanin 1 ameliorates synaptic and behavioral impairments in a mouse model of Alzheimer's disease. The Journal of Neuroscienceeuroscience, 32(44), 15271-15276. https://doi.org/10.1523/JNEUROSCI.2034-12.2012

Milosevic, I. (2018). Revisiting the Role of Clathrin-Mediated Endoytosis in Synaptic Vesicle Recycling. Frontiers in Cellular Neuroscience, 12, 27. https://doi.org/10.3389/fncel.2018.00027

Miosge, L. A., Field, M. A., Sontani, Y., Cho, V., Johnson, S., Palkova, A., ... Andrews, T. D. (2015). Comparison of predicted and actual consequences of missense mutations. Proceedings of the National Academy of Sciences of the United States of America, 112(37), E5189-98. https://doi.org/10.1073/pnas.1511585112

Mutso, M., Morro, A., Smedberg, C., Kasvandik, S., Aquilimeba, M., Teppor, M., ... Varjak, M. (2018). Mutation of CD2AP and SH3KBP1 Binding Motif in Alphavirus nsP3 Hypervariable Domain Results in Attenuated Virus. Viruses, 10(5), 226. https://doi.org/10.3390/v10050226

Ng, P. C., \& Henikoff, S. (2001). Predicting deleterious amino acid substitutions. Genome Research, 11(5), 863-874. https://doi.org/10.1101/gr.176601

Nicolas, G., Charbonnier, C., \& Campion, D. (2016). From Common to Rare Variants: The Genetic Component of Alzheimer Disease. Human Heredity, 81(3), 129-141. https://doi.org/10.1159/000452256

Pierre, A. Saint, \& Genin, E. (2014). How important are rare variants in common disease? Briefings in Functional Genomics, 13(5), 353-361. https://doi.org/10.1093/bfgp/elu025

Raj, T., Li, Y. I., Wong, G., Humphrey, J., Wang, M., Ramdhani, S., ... De Jager, P. L. (2018). Integrative transcriptome analyses of the aging brain implicate altered splicing in Alzheimer's disease susceptibility. Nature Genetics, 50(11), 1584-1592. https://doi.org/10.1038/s41588-018-0238-1

Ramjaun, A. R., Micheva, K. D., Bouchelet, I., \& McPherson, P. S. (1997). Identification and characterization of a nerve terminal-enriched amphiphysin isoform. Journal of Biological Chemistry. https://doi.org/10.1074/jbc.272.26.16700

Reva, B., Antipin, Y., \& Sander, C. (2011). Predicting the functional impact of protein mutations: application to cancer genomics. Nucleic Acids Research, 39(17), e118-e118. https://doi.org/10.1093/nar/gkr407

Rosenthal, S. L., \& Kamboh, M. I. (2014). Late-Onset Alzheimer's Disease Genes and the Potentially Implicated Pathways. Current Genetic Medicine Reports, 2(2), 85-101. https://doi.org/10.1007/s40142-014-0034-x

Saltel, F., Giese, A., Azzi, L., Elatmani, H., Costet, P., Ezzoukhry, Z., ... Jacquemin-Sablon, H. (2017). Unr defines a novel class of nucleoplasmic reticulum involved in mRNA translation. Journal of Cell Science. https://doi.org/10.1242/jcs.198697

Sauna, Z. E., \& Kimchi-Sarfaty, C. (2011). Understanding the contribution of synonymous mutations to human disease. Nature Reviews Genetics, 12(10), 683-691. https://doi.org/10.1038/nrg3051

Shulman, J. M., Imboywa, S., Giagtzoglou, N., Powers, M. P., Hu, Y., Devenport, D., ... Feany,

Peer) reviewing PDF | (2019:03:35860:1:1:NEW 11 Jul 2019) 
682

683

684

685

686

687

688

689

690

691

692

693

694

695

696

697

698

699

700

701

702

703

704

705

706

707

708

709

710

711

712

713

714

715

716

717

718

719

720

721

722

723

724

725

726

727
M. B. (2014). Functional screening in Drosophila identifies Alzheimer's disease susceptibility genes and implicates tau-mediated mechanisms. Human Molecular Genetics, 23(4), 870-877. https://doi.org/10.1093/hmg/ddt478

Stenson, P. D., Mort, M., Ball, E. V., Evans, K., Hayden, M., Heywood, S., ... Cooper, D. N. (2017). The Human Gene Mutation Database: towards a comprehensive repository of inherited mutation data for medical research, genetic diagnosis and next-generation sequencing studies. Human Genetics, 136(6), 665-677. https://doi.org/10.1007/s00439-0171779-6

Tan, S. H., Karri, V., Tay, N. W. R., Chang, K. H., Ah, H. Y., Ng, P. Q., .. Candasamy, M. (2019). Emerging pathways to neurodegeneration: Dissecting the critical molecular mechanisms in Alzheimer's disease, Parkinson's disease. Biomedicine \& Pharmacotherapy, 111, 765-777. https://doi.org/10.1016/j.biopha.2018.12.101

Tebar, F., Bohlander, S. K., \& Sorkin, A. (1999). Clathrin Assembly Lymphoid Myeloid Leukemia (CALM) Protein: Localization in Endocytic-coated Pits, Interactions with Clathrin, and the Impact of Overexpression on Clathrin-mediated Traffic. Molecular Biology of the Cell, 10(8), 2687-2702. https://doi.org/10.1091/mbc.10.8.2687

Treusch, S., Hamamichi, S., Goodman, J. L., Matlack, K. E. S., Chung, C. Y., Baru, V., ... Lindquist, S. (2011). Functional Links Between A-beta Toxicity, Endocytic Trafficking, and Alzheimer's Disease Risk Factors in Yeast. Science. https://doi.org/10.1126/science.1213210

Visscher, P. M., Wray, N. R., Zhang, Q., Sklar, P., McCarthy, M. I., Brown, M. A., \& Yang, J. (2017). 10 Years of GWAS Discovery: Biology, Function, and Translation. The American Journal of Human Genetics, 101(1), 5-22. https://doi.org/10.1016/j.ajhg.2017.06.005

Wang, M., Beckmann, N. D., Roussos, P., Wang, E., Zhou, X., Wang, Q., ... Zhang, B. (2018). The Mount Sinai cohort of large-scale genomic, transcriptomic and proteomic data in Alzheimer's disease. Scientific Data, 5, 180185. https://doi.org/10.1038/sdata.2018.185

Weiner, M. W., Veitch, D. P., Aisen, P. S., Beckett, L. A., Cairns, N. J., Cedarbaum, J., ... Alzheimer's Disease Neuroimaging Initiative. (2015). 2014 Update of the Alzheimer's Disease Neuroimaging Initiative: A review of papers published since its inception. Alzheimer's \& Dementia: The Journal of the Alzheimer's Association, 11(6), e1-120. https://doi.org/10.1016/j.jalz.2014.11.001

Xiao, Q., Gil, S.-C., Yan, P., Wang, Y., Han, S., Gonzales, E., ... Lee, J.-M. (2012). Role of phosphatidylinositol clathrin assembly lymphoid-myeloid leukemia (PICALM) in intracellular amyloid precursor protein (APP) processing and amyloid plaque pathogenesis. The Journal of Biological Chemistry, 287(25), 21279-21289. https://doi.org/10.1074/jbc.M111.338376

$\mathrm{Xu}, \mathrm{D} .$, \& Zhang, Y. (2011). Improving the physical realism and structural accuracy of protein models by a two-step atomic-level energy minimization. Biophysical Journal, 101(10), 2525-2534. https://doi.org/10.1016/j.bpj.2011.10.024

Yang, Y., Faraggi, E., Zhao, H., \& Zhou, Y. (2011). Improving protein fold recognition and template-based modeling by employing probabilistic-based matching between predicted one-dimensional structural properties of query and corresponding native properties of templates. Bioinformatics (Oxford, England), 27(15), 2076-2082. https://doi.org/10.1093/bioinformatics/btr350

Zhang, F., \& Lupski, J. R. (2015). Non-coding genetic variants in human disease. Human Molecular Genetics, 24(R1), R102-R110. https://doi.org/10.1093/hmg/ddv259

Peer] reviewing PDF | (2019:03:35860:1:1:NEW 11 Jul 2019) 
728

Zhang, J., Zheng, X., Yang, X., \& Liao, K. (2009). CIN85 associates with endosomal membrane and binds phosphatidic acid. Cell Research, 19(6), 733-746. https://doi.org/10.1038/cr.2009.51

Zhao, Z., Sagare, A. P., Ma, Q., Halliday, M. R., Kong, P., Kisler, K., ... Zlokovic, B. V. (2016). Central role for PICALM in amyloid-B blood-brain barrier transcytosis and clearance. Nat Neurosci., 18(7), 978-987. https://doi.org/10.1038/nn.4025.Central

Zheng, X., Zhang, J., \& Liao, K. (2014). The basic amino acids in the coiled-coil domain of $\mathrm{CIN} 85$ regulate its interaction with $\mathrm{c}-\mathrm{Cbl}$ and phosphatidic acid during epidermal growth factor receptor (EGFR) endocytosis. BMC Biochemistry, 15(1), 13. https://doi.org/10.1186/1471-2091-15-13

Zhu, L., Zhong, M., Elder, G. A., Sano, M., Holtzman, D. M., Gandy, S., ... Cai, D. (2015). Phospholipid dysregulation contributes to ApoE4-associated cognitive deficits in Alzheimer's disease pathogenesis. Proceedings of the National Academy of Sciences, 112(38), 11965-11970. https://doi.org/10.1073/pnas.1510011112

Zhu, L., Zhong, M., Zhao, J., Rhee, H., Caesar, I., Knight, E. M., ... Cai, D. (2013). Reduction of synaptojanin 1 accelerates A-beta clearance and attenuates cognitive deterioration in an alzheimer mouse model. Journal of Biological Chemistry, 288(44), 32050-32063. https://doi.org/10.1074/jbc.M113.504365 
747

748

749

750

751

752

753

754

755

756

757

758

759

760

761

762

763

764

765

766

767

768

769

\section{Legends}

Figure 1. Structural comparison between the wild type and mutant amino acids of PICALM protein in sticks representation. No addition or deletion of hydrogen bonds between the mutant residues and the surrounding residues were identified in variant (B) rs750147583 (L106S) and (F) rs145115354 (D144N). (D) Variant rs780443419 (F109S) resulted in addition of hydrogen bonds with residues L106. (H) Variant rs765338634 (L179P) resulted in deletion of hydrogen bonds between residues M175 and D176.

Figure 2. Evolutionary conservation of the deleterious nsSNPs of PICALM, SYNJ1, and SH3KBP1 proteins by ConSurf server. (A), (B) and (C) showed the ConSurf output for PICALM, SYNJ1, and SH3KBP1 protein sequences, respectively. The position of each deleterious variant is labeled with a black rectangle.

Figure 3. Superposition of wild type SYNJ1 structure and variant rs781675993 (N200K) mutant structure. This figure shows the truncated protein structure of 100 residues around the mutant sites. Wild type SYNJ1 structure is green, and mutant SYNJ1 structure is blue. Wild type residue $(\mathrm{N})$ at position 200 is red, and mutant residue $(\mathrm{K})$ at position 200 is purple.

Table 1. Variants with altered functional pattern(s) of the 3' and 5'UTRs of PICALM, SYNJ1 and SH3KBP1 genes.

Table 2. List of variants predicted to break or create splice site of PICALM, SYNJ1 and SH3KBP1 genes.

Table 3. Deleterious sSNPs of PICALM and SYNJ1 genes predicted by PredictSNP2.

Table 4. Prediction of PICALM, SYNJ1 and SH3KBP1 deleterious nsSNPs.

Table 5. Total energy deviation and RMSD of deleterious nsSNPs of PICALM, SYNJ1 and SH3KBP1 proteins. 
770 Supplemental data information

771 Supplemental Figure S1. Total number of variants of PICALM, SYNJ1 and SH3KBP1

772 genes.

773 Supplemental Figure S2. Evolutionary conservation of PICALM protein sequence. The 774 result file was generated by ConSurf server.

775 Supplemental Figure S3. Evolutionary conservation of SYNJ1 protein sequence. The result 776 file was generated by ConSurf server.

777 Supplemental Figure S4. Evolutionary conservation of SH3KBP1 protein sequence. The 778 result file was generated by ConSurf server.

779 Supplemental Table S1. Possible consequences of splice site variants predicted by 780 MaxEntScan.

781

782

783

784

785

786

787

788

789

790

791

792

793

794

795

796

Supplemental Table S2. UTR sequences information and the total number of variants of PICALM, SYNJ1 and SH3KBP1 genes.

Supplemental Table S3. Prediction analysis of UTRs variants. S3A-S3C provided the complete prediction result of PICALM, SYNJ1 and SH3KBP1 UTR variants, respectively. S3D showed the list of variants that changed the number of motifs as compare to the respective wild type sequences.

Supplemental Table S4. Prediction analysis of splice site variants. S4A-S4C provided the complete prediction result of PICALM, SYNJ1 and SH3KBP1 splice site variants, respectively. S4D showed the list of variants with score difference exceeding the defined threshold, which were predicted to break or create splice site on the respective genes.

Supplemental Table S5. Prediction analysis of PICALM sSNPs. The result file was generated by PredictSNP2.

Supplemental Table S6. Prediction analysis of SYNJ1 sSNPs. The result file was generated by PredictSNP2.

Supplemental Table S7. Prediction analysis of SH3KBP1 sSNPs. The result file was generated by PredictSNP2. 
797 Supplemental Table S8. Prediction analysis of nsSNPs. S8A-S8C provided the complete 798 prediction result of PICALM, SYNJ1 and SH3KBP1 nsSNPs, respectively. S8D showed the list of 799 variants that were predicted to be the deleterious variants in this study.

800 Supplemental Table S9. The physical and chemical properties of deleterious nsSNPs of 801 PICALM, SYNJ1 and SH3KBP1 proteins.

802

Supplemental Table S10. Prediction of pathogenic and non-pathogenic PSEN1 nsSNPs.

803 Supplemental Table S11. Deleterious variants that were identified in the NGS studies of 804 AD.

805

Supplemental Data S1. Raw data of UTR variants. The information of PICALM, SYNJI and 806 SH3KBP1 UTR variants were retrieved from Ensembl genome database. and $S H 3 K B P 1$ splice site variants were retrieved from Ensembl genome database.

Supplemental Data S3. Raw data of sSNP variants. The information of PICALM, SYNJ1 and SH3KBP1 sSNP variants were retrieved from Ensembl genome database.

811 Supplemental Data S4. Raw data of nsSNP variants. The information of PICALM, SYNJ1 and 812 SH3KBP1 nsSNP variants were retrieved from Ensembl genome database. 


\section{Figure 1}

Structural comparison between the wild type and mutant amino acids of PICALM protein in sticks representation.

No addition or deletion of hydrogen bonds between the mutant residues and the surrounding residues were identified in variant (B) rs750147583 (L106S) and (F) rs145115354 (D144N). (D) Variant rs780443419 (F109S) resulted in addition of hydrogen bonds with residues L106.

(H) Variant rs765338634 (L179P) resulted in deletion of hydrogen bonds between residues M175 and D176. 

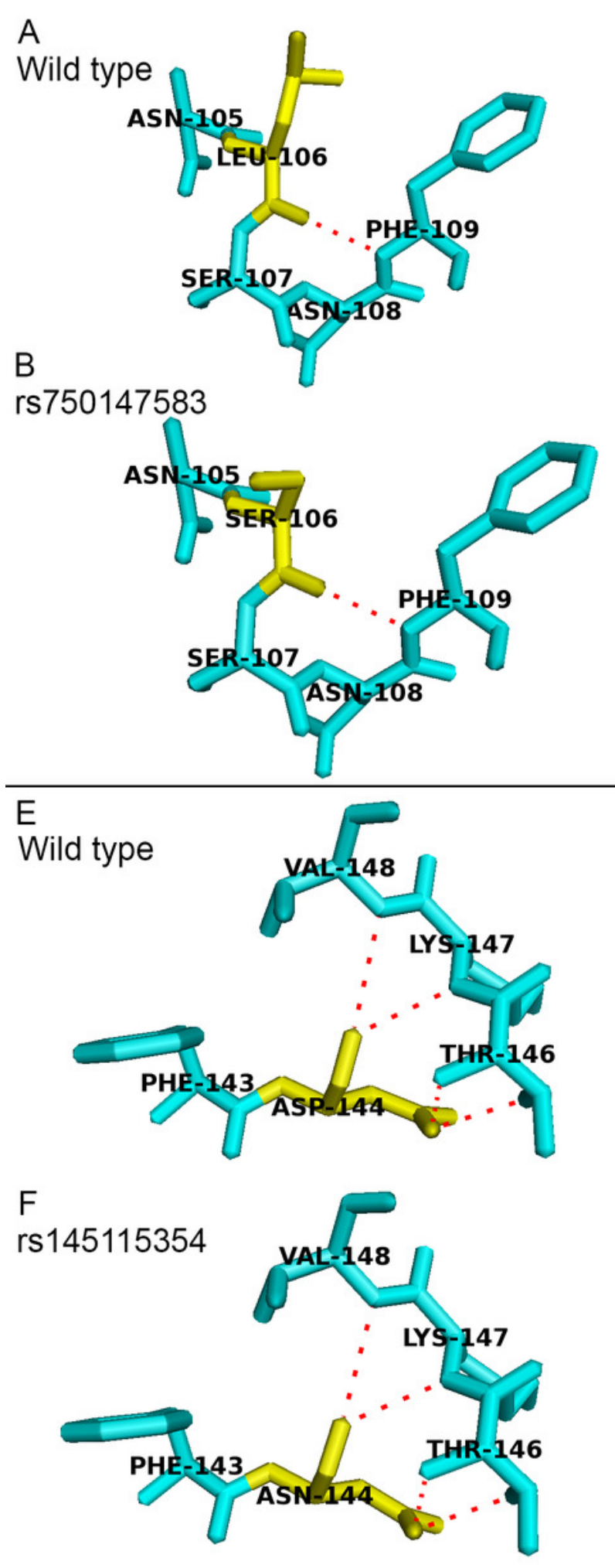

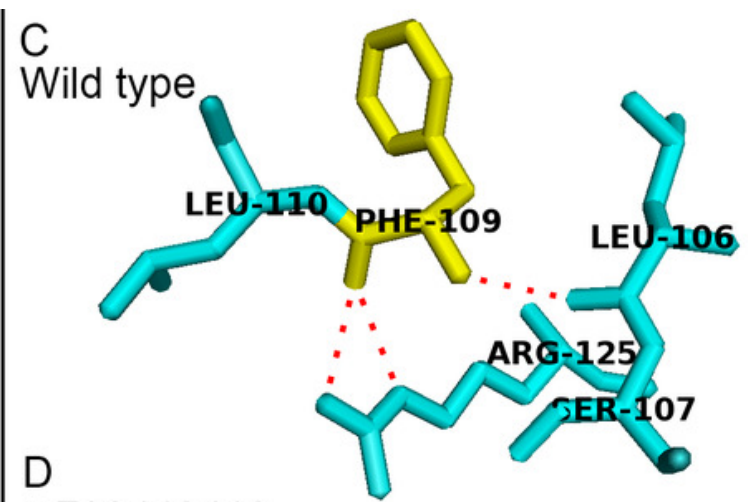

rs780443419

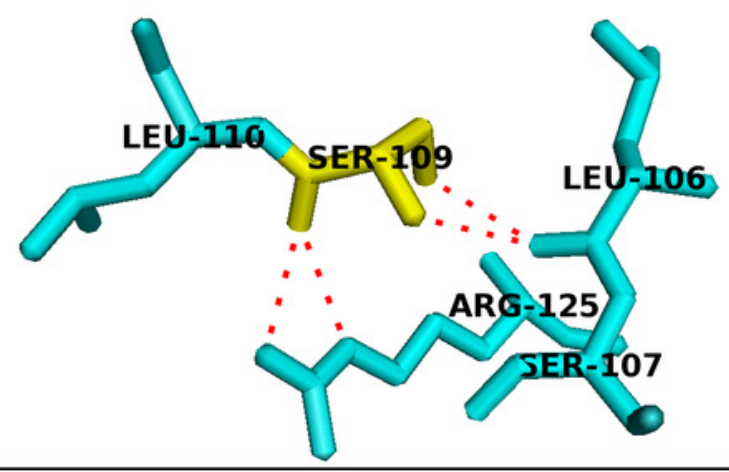

G

Wild type
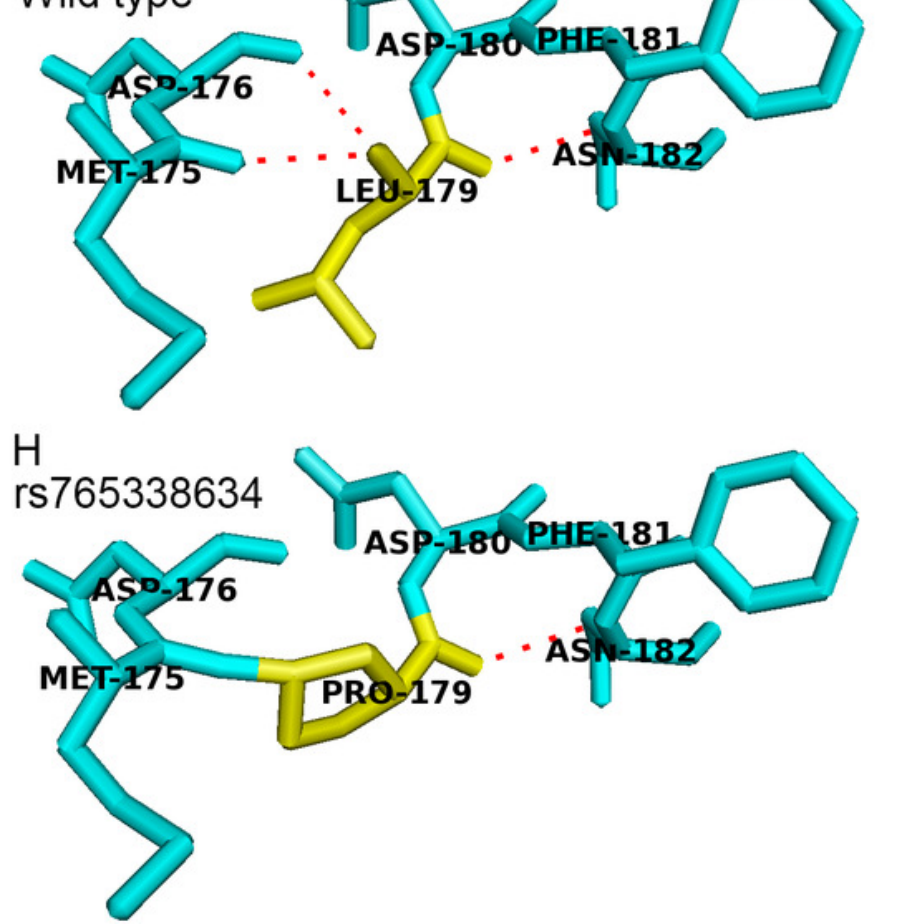
Figure 2

Evolutionary conservation of the deleterious nsSNPS of PICALM, SYNJ1, and SH3KBP1 proteins by ConSurf server.

(A), (B) and (C) showed the ConSurf output for PICALM, SYNJ1, and SH3KBP1 protein sequences, respectively. The position of each deleterious variant is labeled with a black rectangle. 


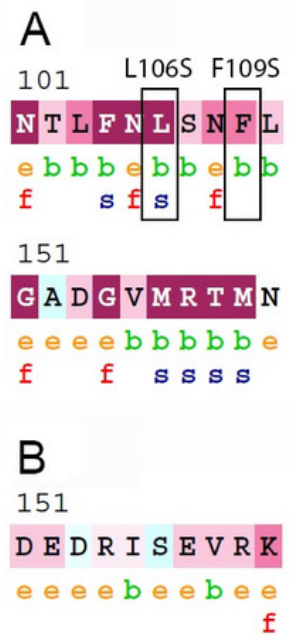

111

DK S G L Q G Y D M

e e e e bebeeb

$f f \quad f \quad f s f s$

161

T E K L L K T V P I

$\mathrm{b} e \mathrm{e} \mathrm{b} \mathrm{b} e \mathrm{e} \mathrm{b} e \mathrm{~b}$

f s s f $f \quad f$
121 S T F I R R Y S R Y

$\mathrm{b} b \mathrm{~b} b \mathrm{~b} e \mathrm{~b} \mathrm{~b} e \mathrm{e}$

s s sfs $\mathrm{s}$

171

L179P

131

$141^{D 144 N}$

L N E K A V S Y R Q

VA D F T K V R

L $b$ b e be

$b$ b b e b b e b e e

fff $\mathrm{s}$ f $\mathrm{s} \quad \mathrm{s}$ f s s $\mathrm{f}$ sf

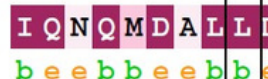

F N V N S N E L T N

191
161

V L N S G N F F A

$\mathrm{b} b$ b e b b b b b b

$f \mathrm{f} s \mathbf{s} s$

$251 \quad \mathrm{R} 258 \mathrm{Q}$

A G T R N V R G T

$\mathrm{b}$ e $\mathrm{b}$ e b e b e b e

$s$ f $s$ s $s f s$

301

L Q V G S H R V R M

e e b b e e e be b

$\mathrm{f} s \mathrm{~s} f \mathrm{f}$

401

G F F Y F N G S E V

$e \mathrm{~b} b \mathrm{~b} e e e e e$

451

A E K P Q L V T R F

e e e e e b b e e b f

f $\mathrm{s}$

501

T I QN N F D S S

$\mathrm{b} b$ b e e b e e e b

s sffif

601

L L D A P K L A G I

$\mathrm{b} e \mathrm{e} b$ e e e e e e

$s \mathrm{f} f$

701

M G G A T N K G A

e e e e e e e e b

ffff $f$ f $f$

751

S F P M R M L F S

$\mathrm{b} b$ e b b e b b b b

s $f \quad f$

f

C

601

PAASS QA A E

261

NDDGHVAN F V

e e e e e b bebb

ff $f$ s sfs s

311

SR G F E A N A P A

$\mathrm{b}$ e e b e b e b e b

$$
f \mathrm{f} f \mathrm{~s}
$$

411 Q414R

Q R C Q S G T V R T

e e e $\begin{array}{ccc}e & e \in b & b \\ f & f & f\end{array}$

461

Q EV F R S M W V

e e b b e b b b b e

$$
\text { f s s }
$$

$511 \quad$ I515T

K Q E A I D V L L L e e e b b $b \vec{b} b \vec{b}$

611

Q E F Q D K R K P

ee e e e e e e

711

VAIRMLF H T T

$\mathrm{b} b \mathrm{~b} b \mathrm{~b}$ b b b b b

s s

761 H D Y V F W C G D F

$e=b \mathrm{~b} b \mathrm{~b} b \mathrm{~b} \in \mathrm{b}$

f $f \quad s$ s s $\mathrm{s}$ s
171

W S A S G I S L D L

$\mathrm{b} e \mathrm{e} e \mathrm{e} e \mathrm{~b} e$

f

271

E T E QVVY L D D

e bee b b b bee

$\mathrm{f} s \mathrm{f} f$

321

f

F D R H F T L K N

$\mathrm{b} e \mathrm{e} \mathrm{b} \mathrm{b} \in \mathrm{b} \mathrm{b} e \mathrm{e}$

$$
\text { f s }
$$

421

N C L D C L D R T N

$e \mathrm{~b} b$ e b b e e e e

f s $s$ ssffff

471

N G D S I S K I Y A

e e e e b b e b b b

fff $f$ s $\quad s$ $\mathrm{f} f \mathrm{~s}$ s

521

GN T L N S D L A D

$\mathrm{b} e \mathrm{~b} b$ e e e b e e

s $f \quad f f$

$621 \quad$ G627S 631

T D I F I G F E E

$\mathrm{b} e \mathrm{~b} b \mathrm{~b} b \mathrm{~b} b=e$

f $\quad \mathrm{s} s \mathrm{~s}$

C723G

S C FVCSHFA

$\mathrm{b} b \mathrm{~b} b \mathrm{~b} b \mathrm{~b} \mathrm{~b} \mathrm{~b} e$

771 L776S

181

191

N200R

SLNAHRSMQE QTT DNRFFWN

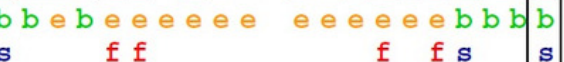

281 R289Q 291

SVSSFIQIRG SVPLFWEPG

e b b b b beb eb ebeb b beeee

$331 \quad$ V338A 341

L Y GK Q I IVNL L G S KE EH M L

e e eeeb b b b b b beeeeeeb b

ff $f \quad \square s \quad s \quad$ f f f f s

431 G437D 441

SVQAF L G EM LAKQLEALGL

$\mathrm{b} b$ b b b b b b b bee e beebee s f

481

G487R

G T G A L E G K A K

$b e e b b=e$ e

$s f f \quad f f \quad f$

$531 \quad 541$

KARALLTTGS LRVSEQTLQS

$e b=b b b e e b e b=b b e e b b e e$

$631 \quad 641$

MVELNAGNIV SAST TNQKLW

$\mathrm{b} b$ e b e b b b b b b b e e e e e e b b s sfifs s

731

f $f f f$ s

A G QSQVKERN

e e e e beeee

$\mathrm{f} f \mathrm{fff} \quad \mathrm{f} f \mathrm{f}$

781

V K E L I R Q Q W

$\mathrm{b} e \mathrm{e} b \mathrm{~b}$ e e e e b

f

$741 \quad$ 1746T

E D F I E I A R K L e e b e e b b e e b

791

D S L I A G D Q L I

$e$ e $b e e b e e b e$

$f \mathrm{fs}$

e e e e e e b b e

611

621

E L R T Q V R E L R

$\begin{array}{ll}b & e \\ s & f\end{array}$
S I I E T M K Q Q

e b b e e b e e e e

f $f \mathrm{f}$
631

K R EIKQLLS E

$e e e b e e b b e e$

f $\quad \mathrm{s}$
641 R648Q

L D E EKK I $R$ L

$\begin{array}{lll}\mathbf{s} f \boldsymbol{f} & \mathrm{f}\end{array}$ 


\section{Figure 3}

Superposition of wild type SYNJ1 structure and variant rs781675993 (N200K) mutant structure.

This figure shows the truncated protein structure of 100 residues around the mutant sites. Wild type SYNJ1 structure is green, and mutant SYNJ1 structure is blue. Wild type residue (N) at position 200 is red, and mutant residue (K) at position 200 is purple.

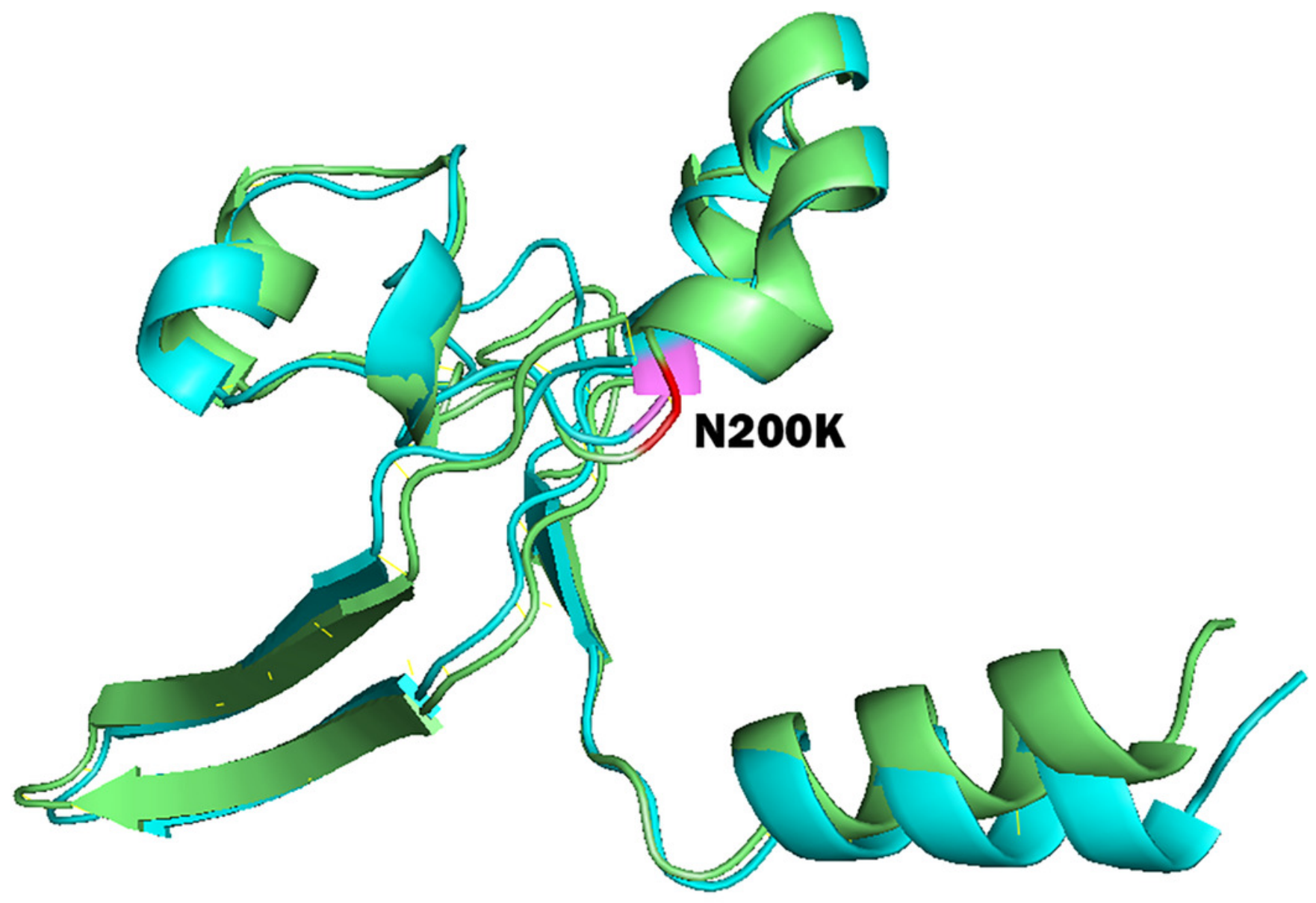




\section{Table $\mathbf{1}$ (on next page)}

Variants with altered functional pattern(s) of the UTRs of PICALM, SYNJ1 and SH3KBPI genes. 
1

2

Table 1. Variants with altered functional pattern(s) of the UTRs of PICALM, SYNJ1 and SH3KBP1 genes.

\begin{tabular}{|c|c|c|c|c|c|}
\hline \multirow[t]{2}{*}{ Variants ID } & \multirow[t]{2}{*}{ Ref/Alt } & \multirow{2}{*}{$\begin{array}{l}\text { Minor Allele } \\
\text { Frequency }\end{array}$} & \multicolumn{2}{|c|}{$\begin{array}{l}\text { Number of } \\
\text { motif }\end{array}$} & \multirow[t]{2}{*}{ Remarks } \\
\hline & & & 3 ' & 5 & \\
\hline \multicolumn{6}{|l|}{ PICALM } \\
\hline Wild type & & & 10 & 1 & \\
\hline rs796143140 & $\mathrm{T} / \mathrm{C}$ & NA & 11 & 1 & Addition of K-box at position 212-219 \\
\hline \multicolumn{6}{|l|}{ SYNJ1 } \\
\hline Wild type & & & 20 & - & \\
\hline rs777138831 & $\mathrm{G} / \mathrm{C}$ & NA & 21 & - & Addition of uORF at position 1915-1995 \\
\hline rs147590143 & $\mathrm{G} / \mathrm{T}$ & NA & 21 & - & Addition of uORF at position 1915-1995 \\
\hline rs780084162 & $\mathrm{G} / \mathrm{T}$ & NA & 21 & - & Addition of UNR-bs at position $1037-1047$ \\
\hline rs767649429 & $-/ \mathrm{AA}$ & NA & 19 & - & Deletion of uORF at position $942-1037$ \\
\hline rs 143739621 & $\mathrm{G} / \mathrm{A}$ & $0.00002 / 2$ & 19 & - & Deletion of uORF at position $942-1037$ \\
\hline rs111516740 & $\mathrm{G} / \mathrm{A}$ & $0.0004 / 54$ & 21 & - & Addition of uORF at position $887-979$ \\
\hline rs761852713 & $-/ \mathrm{G}$ & $0.0001 / 7$ & 17 & - & $\begin{array}{l}\text { Deletion of uORFs at position 372-437, } \\
464-535 \text { and } 644-751\end{array}$ \\
\hline rs79652470 & $\mathrm{C} / \mathrm{T}$ & $0.0001 / 13$ & 17 & - & $\begin{array}{l}\text { Deletion of uORFs at position } 182-289 \text {, } \\
372-437,464-535,644-751 \text {, addition of } \\
\text { uORF at position } 205-810\end{array}$ \\
\hline \multicolumn{6}{|l|}{ SH3KBP1 } \\
\hline Wild type & & & 19 & 2 & \\
\hline rs778448080 & $\mathrm{G} / \mathrm{T}$ & NA & 18 & 2 & Deletion of GY-box at position 2064-2070 \\
\hline rs192424738 & $\mathrm{C} / \mathrm{T}$ & NA & 20 & 2 & Addition of uORF at position 1380-1469 \\
\hline rs755895016 & $\mathrm{A} /-$ & NA & 18 & 2 & Deletion of uORF at position $1299-1370$ \\
\hline
\end{tabular}

3 


\section{Table 2 (on next page)}

List of variants predicted to break or create splice site of PICALM, SYNJ1 and SH3KBPI genes. 
Table 2. List of variants predicted to break or create splice site of PICALM, SYNJ1 and SH3KBP1 genes.

\begin{tabular}{|c|c|c|c|c|c|c|c|}
\hline Variants ID & Ref/Alt & $\begin{array}{l}\text { Minor Allele } \\
\text { Frequency }\end{array}$ & Exon & Intron & $\begin{array}{c}\text { MaxEntScan } \\
\text { Ref }\end{array}$ & $\begin{array}{c}\text { MaxEntScan } \\
\text { Alt }\end{array}$ & $\begin{array}{c}\text { Score difference } \\
(\%)\end{array}$ \\
\hline \multicolumn{8}{|l|}{ PICALM } \\
\hline rs371001564 & $\mathrm{G} / \mathrm{A}$ & $0.00002 / 2$ & $15 / 20$ & & 9.065917 & 0.623344 & -93.12 \\
\hline rs781144984 & $\mathrm{T} / \mathrm{C}$ & $0.000008 / 1$ & $16 / 20$ & & 7.971843 & 2.847240 & -64.28 \\
\hline rs768239913 & $\mathrm{A} / \mathrm{G}$ & $0.00007 / 8$ & $18 / 20$ & & 8.542122 & 2.867856 & -66.43 \\
\hline \multicolumn{8}{|l|}{ SYNJ1 } \\
\hline rs779813770 & $\mathrm{C} / \mathrm{A}$ & $0.00009 / 1$ & & $1 / 32$ & 8.703310 & 0.199354 & -97.71 \\
\hline rs376825246 & $\mathrm{T} / \mathrm{C}$ & $0.000008 / 1$ & & $11 / 32$ & 7.844178 & 3.885060 & -50.47 \\
\hline rs756697570 & $\mathrm{C} / \mathrm{T}$ & $0.00005 / 6$ & $12 / 33$ & & 7.638176 & 5.268185 & -31.03 \\
\hline rs748110438 & $\mathrm{G} / \mathrm{T}$ & $0.00003 / 3$ & & $13 / 32$ & 8.681559 & 0.239421 & -97.24 \\
\hline rs367718431 & $\mathrm{T} / \mathrm{G}$ & $0.000009 / 1$ & & $13 / 32$ & 8.681559 & 5.646822 & -34.96 \\
\hline rs367718431 & $\mathrm{T} / \mathrm{C}$ & $0.000009 / 1$ & & $13 / 32$ & 8.681559 & 4.215421 & -51.44 \\
\hline rs758644105 & $\mathrm{G} / \mathrm{T}$ & $0.000008 / 1$ & & $16 / 32$ & 8.941208 & 0.759180 & -91.51 \\
\hline rs746368700 & $\mathrm{A} / \mathrm{T}$ & $0.000009 / 1$ & $17 / 33$ & & 9.256318 & 4.683645 & -49.40 \\
\hline rs538934316 & $\mathrm{C} / \mathrm{T}$ & $0.00003 / 4$ & & $19 / 32$ & 5.263433 & -3.346463 & -163.58 \\
\hline rs755765674 & $\mathrm{A} / \mathrm{C}$ & $0.00002 / 2$ & $23 / 33$ & & 6.505994 & 3.822270 & -41.25 \\
\hline rs533064963 & $\mathrm{T} / \mathrm{A}$ & $0.00002 / 3$ & & $25 / 32$ & 2.515213 & 3.906733 & +55.32 \\
\hline rs61752550 & $\mathrm{G} / \mathrm{A}$ & $0.0001 / 11$ & $29 / 33$ & & 9.393973 & 3.084793 & -67.16 \\
\hline rs373831795 & $\mathrm{T} / \mathrm{A}$ & NA & & $31 / 32$ & 10.077445 & 1.573490 & -84.39 \\
\hline
\end{tabular}


Table 2. List of variants predicted to break or create splice site of PICALM, SYNJ1 and SH3KBP1 genes (continued).

\begin{tabular}{c|c|c|c|c|c|c|c}
\hline Variants ID & Ref/Alt & $\begin{array}{c}\text { Minor Allele } \\
\text { Frequency }\end{array}$ & Exon & Intron & $\begin{array}{c}\text { MaxEntScan } \\
\text { Ref }\end{array}$ & $\begin{array}{c}\text { MaxEntScan } \\
\text { Alt }\end{array}$ & $\begin{array}{c}\text { Score difference } \\
(\%)\end{array}$ \\
\hline SH3KBP1 & & & & & & & \\
\hline rs777396528 & T/C & $0.00001 / 1$ & & $8 / 17$ & 8.138004 & 4.452207 & -45.29 \\
\hline rs61761898 & G/C & $0.0035 / 303$ & $15 / 18$ & & 9.095315 & 5.850377 & -35.68 \\
\hline
\end{tabular}


Table 3 (on next page)

Deleterious SSNPS of PICALM and SYNJ1 genes predicted by PredictSNP2. 
Table 3. Deleterious sSNPs of PICALM and SYNJ1 genes predicted by PredictSNP2.

\begin{tabular}{c|c|c|c|c|c|c|c|c}
\hline Variants ID & Ref/Alt & $\begin{array}{c}\text { Minor Allele } \\
\text { Frequency }\end{array}$ & CADD & DANN & FATHMM & FunSeq2 & GWAVA & $\begin{array}{c}\text { Predict } \\
\text { SNP2 }\end{array}$ \\
\hline $\boldsymbol{P I C A L M}$ & & & & & & & & \\
\hline rs779752380 & $\mathrm{C} / \mathrm{G}$ & $0.000010 / 1$ & 15.54 & 0.947688 & 0.91404 & 4 & 0.37 & 0.549842 \\
\hline rs777199260 & $\mathrm{A} / \mathrm{G}$ & $0.000008 / 1$ & 8.879 & 0.869109 & 0.93421 & 4 & 0.42 & 0.091749 \\
\hline rs775830584 & T/C & NA & 13.32 & 0.963935 & 0.21828 & 3 & 0.32 & 0.111345 \\
\hline rs759002840 & G/A & $0.0002 / 20$ & 17.47 & 0.964966 & 0.79634 & 0 & 0.34 & 0.459234 \\
\hline rs367839126 & G/A & $0.00003 / 3$ & 19.81 & 0.959689 & 0.889 & 0 & 0.41 & 0.485786 \\
\hline $\boldsymbol{S Y N J 1}$ & & & & & & & & 0.58 \\
\hline rs768565296 & C/T & $0.000010 / 1$ & 17.88 & 0.91747 & 0.91944 & 0 & 0.473949 \\
\hline rs869206401 & G/A & NA & 22.4 & 0.934833 & 0.75779 & 0 & 0.51 & 0.028194 \\
\hline rs772641102 & G/A & NA & 21.4 & 0.942502 & 0.36483 & 0 & 0.41 & 0.019858 \\
\hline
\end{tabular}


Table 4 (on next page)

Prediction of PICALM, SYNJ1 and SH3KBP1 deleterious nSSNPs. 
Table 4. Prediction of PICALM, SYNJ1 and SH3KBP1 deleterious nsSNPs.

\begin{tabular}{|c|c|c|c|c|c|c|c|}
\hline Variant ID & AA Subs & $\begin{array}{c}\text { Minor Allele } \\
\text { Frequency }\end{array}$ & SIFT score & $\begin{array}{l}\text { PolyPhen } \\
\text { PSIC score }\end{array}$ & $\begin{array}{c}\text { Mutation } \\
\text { Assessor }\end{array}$ & $\begin{array}{c}\text { I-Mutant } \\
\text { DDG }\end{array}$ & SNPs\&GO \\
\hline \multicolumn{8}{|l|}{ PICALM } \\
\hline rs 750147583 & L106S & NA & 0 & 0.999 & Medium & -1.93 & 0.644 \\
\hline rs780443419 & F109S & 0.00001 & 0 & 0.994 & Medium & -2.81 & 0.794 \\
\hline rs145115354 & D144N & 0.000008 & 0 & 1 & Medium & -1.57 & 0.784 \\
\hline rs765338634 & L179P & 0.00001 & 0 & 0.989 & Medium & -2.29 & 0.692 \\
\hline \multicolumn{8}{|l|}{ SYNJ1 } \\
\hline rs781675993 & N200K & 0.000008 & 0 & 0.99 & High & -1.34 & 0.735 \\
\hline rs398122403 & $\mathrm{R} 258 \mathrm{Q}$ & 0.00002 & 0 & 0.978 & High & -1.23 & 0.841 \\
\hline rs762909719 & R289Q & 0.000009 & 0 & 1 & High & -1.77 & 0.723 \\
\hline rs771755243 & V338A & 0.000008 & 0 & 0.928 & Medium & -1.16 & 0.598 \\
\hline rs768897710 & Q414R & 0.000008 & 0 & 0.999 & High & -1.14 & 0.751 \\
\hline rs779479360 & G437D & 0.00002 & 0 & 0.939 & Medium & -1.42 & 0.736 \\
\hline rs775515863 & G487R & 0.000008 & 0 & 0.988 & Medium & -1.81 & 0.79 \\
\hline rs752563697 & G494D & 0.000008 & 0 & 0.943 & High & -1.74 & 0.632 \\
\hline rs771070426 & I515T & 0.000008 & 0 & 0.969 & Medium & -3.5 & 0.653 \\
\hline rs756845805 & G627S & 0.000008 & 0 & 0.999 & Medium & -1.6 & 0.751 \\
\hline rs751110096 & $\mathrm{C} 723 \mathrm{G}$ & 0.000008 & 0 & 0.996 & Medium & -2.59 & 0.826 \\
\hline rs147929290 & $\mathrm{I} 746 \mathrm{~T}$ & 0.000008 & 0.03 & 0.951 & Medium & -2.42 & 0.633 \\
\hline rs745418083 & L776P & 0.000008 & 0 & 0.981 & Medium & -2.32 & 0.861 \\
\hline
\end{tabular}


Table 4. Prediction of PICALM, SYNJ1 and $S H 3 K B P 1$ deleterious nsSNPs (continued).

\begin{tabular}{c|c|c|c|c|c|c|c}
\hline Variant ID & AA Subs & $\begin{array}{c}\text { Minor Allele } \\
\text { Frequency }\end{array}$ & SIFT score & $\begin{array}{c}\text { PolyPhen } \\
\text { PSIC score }\end{array}$ & $\begin{array}{c}\text { Mutation } \\
\text { Assessor }\end{array}$ & $\begin{array}{c}\text { I-Mutant } \\
\text { DDG }\end{array}$ & $\begin{array}{c}\text { SNPs\&GO } \\
\text { SH3KBP1 }\end{array}$ \\
\hline rs770229859 & R648Q & NA & 0 & 0.951 & Medium & -1.07 & 0.658 \\
\hline
\end{tabular}




\section{Table 5 (on next page)}

Total energy deviation and RMSD of deleterious nsSNPS of PICALM, SYNJ1 and SH3KBP1 proteins. 
Table 5. Total energy deviation and RMSD of deleterious nsSNPs of 2 PICALM, SYNJ1 and SH3KBP1 proteins.

\begin{tabular}{|c|c|c|c|c|}
\hline Variant ID & AA Subs. & Total Energy (kJ/mol) & Energy difference & $\operatorname{RMSD}(\AA)$ \\
\hline PICALM (wild type) & & -24238.746 & & \\
\hline rs750147583 & L106S & -24759.834 & -521.088 & 1.26 \\
\hline rs780443419 & F109S & -24921.291 & -682.545 & 1.45 \\
\hline rs145115354 & $\mathrm{D} 144 \mathrm{~N}$ & -24494.619 & -255.873 & 1.45 \\
\hline rs 765338634 & L179P & -24824.025 & -585.279 & 1.13 \\
\hline SYNJ1 (wild type) & & -19307.051 & & \\
\hline rs 781675993 & $\mathrm{~N} 200 \mathrm{~K}$ & -11031.447 & +8275.604 & 2.34 \\
\hline rs398122403 & R258Q & 3057.503 & +22364.554 & 2.15 \\
\hline rs762909719 & R289Q & -21897.707 & -2590.656 & 2.02 \\
\hline rs 771755243 & V338A & -17907.066 & +1399.985 & 2.09 \\
\hline rs768897710 & Q414R & -18169.162 & +1137.889 & 2.04 \\
\hline rs779479360 & G437D & -19518.217 & -211.166 & 2.08 \\
\hline rs775515863 & G487R & -28591.270 & -9284.219 & 2.1 \\
\hline rs752563697 & G494D & -6042.169 & +13264.882 & 2.24 \\
\hline rs771070426 & I515T & -9617.264 & +9689.787 & 2.13 \\
\hline rs 756845805 & G627S & -25429.416 & -6122.365 & 2.13 \\
\hline rs751110096 & C723G & -23460.332 & -4153.281 & 1.83 \\
\hline rs147929290 & I746T & -20893.076 & -1586.025 & 2.25 \\
\hline rs 745418083 & L776P & -18299.359 & +1007.692 & 2.14 \\
\hline SH3KBP1 (wild type) & & -15365.350 & & \\
\hline rs770229859 & R648Q & -16167.838 & -802.488 & 1.88 \\
\hline
\end{tabular}

